\title{
C-MYC inducible onco-IncRNA LINC00036 acting as EGFR mRNA stabilizer via RNA-protein and RNA- RNA interactions decreases the sensitivity of gefitinib in human cancer
}

\section{Shouping Xu}

Harbin Medical University Third Clinical College: Tumor Hospital of Harbin Medical University

\section{Lin Wan}

Harbin Medical University Third Clinical College: Tumor Hospital of Harbin Medical University

\section{Qin Wang}

Harbin Medical University Third Clinical College: Tumor Hospital of Harbin Medical University Huizi Yin

Harbin Medical University Third Clinical College: Tumor Hospital of Harbin Medical University Kun Qiao

Harbin Medical University Third Clinical College: Tumor Hospital of Harbin Medical University

Siwei Li

Harbin Medical University Third Clinical College: Tumor Hospital of Harbin Medical University

Jinfeng Zhang

Harbin Medical University Third Hospital: Tumor Hospital of Harbin Medical University

\section{Hao Wu}

Harbin Medical University Third Clinical College: Tumor Hospital of Harbin Medical University

\section{Meiying Shen}

Harbin Medical University Third Clinical College: Tumor Hospital of Harbin Medical University

\section{Shipeng Ning}

Harbin Medical University Third Clinical College: Tumor Hospital of Harbin Medical University

Da Pang ( $\sim$ pangda@ems.hrbmu.edu.cn )

Tumor Hospital of Harbin Medical University

\section{Research}

Keywords: LINC00036, c-MYC, EGFR, Competing endogenous RNAs, Drug sensitivity, Target therapy

Posted Date: February 25th, 2021

DOI: https://doi.org/10.21203/rs.3.rs-240846/v1 
License: (c) (i) This work is licensed under a Creative Commons Attribution 4.0 International License. Read Full License 


\section{Abstract}

Background: The oncogenic IncRNA based strategies for combating cancer may usher in a new and promising paradigm in cancer therapy. However, few studies have been performed to solve such a critical issue. The complex traits and molecular mechanism of such IncRNAs in tumorigenesis and their relationship with sensitivity of gefitinib in human cancer have not been investigated.

Methods: We aimed to identify and validate such a novel oncogenic LINC00036 using transcriptome sequencing approach and a large number of tissue samples of different types of cancer from the our cancer center cohort and public data cohorts from the Cancer Genome Atlas $₫$ Gene Expression Omnibus and Cancer Cell Line Encyclopedia. Moreover, series of in vitro and in vivo experiments were performed to examine its roles in tumorigenesis and the sensitivity of gefitinib in different types of cancer cells. Special nanoparticle via a more potent delivery system was developed to investigate the feasibility of targeting LINC00036 in vivo. Furthermore, chromatin immunoprecipitation (ChIP)-sequencing, ChIP, actinomycin D assay, dual-luciferase reporter assay, RNA pull-down and RNA immunoprecipitation were performed were developed to uncover the molecular mechanism.

Results: LINC00036 that associated with poor prognosis is significantly upregulated in human cancer tissues. Series of in vitro and in vivo experiments reveal that LINC00036 promotes tumorigenesis and decreases the sensitivity of gefitinib in different types of cancer cells. LINC00036 targeting nanoparticle markedly reduced the growth of human cancer xenografts. Mechanistically, LINC00036 is a direct transcriptional target of c-MYC and a positive feedback loop of the c-MYC-LINC00036-EGFR axis exists in human cancer. LINC00036 acts as an EGFR mRNA stabilizer via RNA-protein and RNA-RNA interactions, inducing the hyper-activation of the downstream AKT and MAPK signaling pathways, which in turn decreases the sensitivity of gefitinib in human cancer.

Conclusions: LINC00036, a c-MYC inducible onco-IncRNA, acts an oncogene in human cancer and decreases the sensitivity of gefitinib through positive feedback loop of the c-MYC-LINC00036-EGFR axis. Overall, this study broadens knowledge regarding novel onco-IncRNAs and will assist in developing feasible onco-IncRNAs based-targeted therapeutic strategies to improve the sensitivity of gefitinib in human cancer.

\section{Background}

Currently, the ENCODE project indicates the existence of almost 28,000 long noncoding RNAs (IncRNAs, > 200 nucleotides in length), many of which are still being identified and are yet to be annotated or characterized [1, 2]. Furthermore, genome-wide evidence indicates that many IncRNAs exhibit cancerspecific expression and act as oncogenes by regulating gene expression at the epigenetic, transcriptional, and post-transcriptional level $[3,4]$. LncRNAs possess many attractive properties that enable their use as potential therapeutics for human cancer [5-7]. LncRNAs possess many attractive properties that enable their use as potential therapeutics for human cancer [8]. Importantly, IncRNAs can be targeted via multiple 
mechanisms with diverse modes of action, including 1 ) inactivation by small interfering (si)RNAs and/or antisense oligonucleotides (ASOs) [9, 10], aptamers, and ribozymes; 2) modulation by altering the IncRNA-encoding promoter activity [11]; and 3) blocking the interactions between IncRNAs and regulatory interaction sites using factors (such as small synthetic molecules/peptides) or aptamers [12]. In addition, the large number of regulatory interaction sites in IncRNAs provides a platform for actively pursuing novel oncogenic IncRNA (onco-IncRNA)-based therapeutic strategies against cancer [13, 14].

Although the Food and Drug Administration of USA has recently approved the use of several RNA-based therapeutics for infectious diseases (such as hepatitis $C$ virus and human immunodeficiency virus infections) and rare diseases (polyneuropathy), the utility of onco-IncRNAs is still being evaluated in large pre-clinical or early-stage clinical trials [15]. Antisense oligonucleotides-mediated inhibition of MALAT1 reduces cancer progression [16-18] and metastasis in breast tumors [19]. Apart from direct anti-cancer therapy, onco-IncRNAs can also be considered for combination therapy to improve the specificity, sensitivity, and therapeutic efficacy of existing anti-cancer therapeutic drugs [20-22]. Knockdown of the IncRNA HOTAIR increased sensitivity of patients with head and neck squamous cell cancer to platinum therapy [23]. The field of IncRNA therapeutics is currently undergoing a renaissance with advancements in chemotherapy, radiotherapy, and immunotherapy for various malignancies [24-27]. Hence, it is evident that targeting onco-IncRNA might be used for tumor therapy and can improve the specificity, sensitivity and therapeutic effect of existing anti-cancer therapeutic drugs. However, considering the large number of aberrantly expressed IncRNAs in various cancers, identification of the most fundamental onco-IncRNAs with broad anticancer activity remains highly challenging.

Epidermal growth factor receptor (EGFR) is a transmembrane receptor tyrosine kinase protein, and constitutive activation of EGFR has been shown connected with tumorigenesis, progression and unfavorable prognosis in human cancers $[28,29]$. Activation of EGFR results in ignition of downstream signaling pathways, such as phosphatidylinositol 3-kinase/serine threonine protein kinase (PI3K/Akt) and phosphorylating mitogen-activated protein kinase (MAPK) pathways [30-32], which play major roles in the proliferation, survival, drug sensitivity and resistance in many different cell types [33,34]. Clinical trials have verified that novel therapeutic regimens targeting EGFR including monoclonal antibodies, tyrosine kinase inhibitors, phosphatidylinositol 3-kinase (PI3K) inhibitors and antisense gene therapy, have become one of the most widely studied therapeutic targets in human cancers [35-37]. Gefitinib is a firstgeneration tyrosine kinase inhibitor that binds to the kinase domain of EGFR, preventing its phosphorylation and blocking downstream signaling cascades and is approved for the first-line treatment of patients with EGFR mutation-positive metastatic non-small cell lung cancer [38]. However, the therapeutic efficiency of single-agent gefitinib treatment is variable [39, 40]. The reduction of gefitinib sensitivity is one of the main obstacles and one of the important reasons leading to unfavorable prognosis. Many view co-targeting therapies as an important next step to improve the clinical outcome of cancer patient [38, 41, 42]. Currently, onco-IncRNAs have attracted a lot of attention as potential targets of drug-sensitivity in cancers due to their functions in cell proliferation, metastasis, and epithelialmesenchymal transition. In this study, we focused on the role of onco-IncRNAs in drug-sensitivity. 
Hence, the aim of this study was to identify novel onco-IncRNAs associated with sensitivity of gefitinib and develop feasible onco-IncRNA-targeted therapeutic strategies against cancer. In this current study, LINC00036 was identified as a novel broad spectrum and potent cancer propellant via highthroughput transcriptome sequencing and further validation in human cancer samples from the Harbin Medical University Cancer Center (HMUCC) and public data cohorts. We further demonstrate an uncharacterized positive feedbck loop of the c-MYC-LINC00036-EGFR axis and conclude that LINC00036 acts as an EGFR mRNA stabilizer via both RNA-protein and RNA-RNA interactions, inducing the hyperactivation of the downstream AKT and MAPK signaling pathways, which in turn reciprocally enhances cMYC transcription, and potentiates the receptor tyrosine kinase cascade during tumorigenesis and decreases sensitivity of gefitinib. It is noteworthy that targeting of LINC00036 represents a promising IncRNA-based therapeutic strategy for combating cancer and may usher in a new paradigm for next generation anti-cancer therapy.

\section{Materials And Methods}

\section{Clinical sample collection}

The 185 breast cancer tissues, 36 lung cancer, 32 glioma, and 21 gastric cancer tissues and their corresponding normal tissues were obtained from HMUCC. Eligible patients with a histological diagnosis of cancer who had received neither chemotherapy nor radiotherapy before surgical resection were recruited to the present study. All samples were frozen in liquid nitrogen immediately after surgical resection, and only tumors with $>80 \%$ tumor cells were selected for RNA extraction. Two independent senior pathologists confirmed the pathological diagnosis and molecular subtype of each cancer tissue. This study conformed to the clinical research guidelines and was approved by the research ethics committee of the Harbin Medical University Cancer Center. We obtained written informed consent from all patients.

\section{Cell line}

The human breast cancer cell lines (MCF10A, UACC-812, Hs578T, HCC70, MCF-7, MDA-MB-231, MDA-MB453, MDA-MB-468 and T-47D), the human gastric carcinoma cell lines (SGC-7901 and MKN-45), the human lung cancer cell lines ( $\mathrm{NCl}-\mathrm{H} 1975$ and $\mathrm{NCl}-\mathrm{H} 1650)$, the human kidney cancer cell line (786-0), the human liver cancer cell line (Hep3B), the human glioma cell line (U251), the human ovarian cancer cell line (SKOV-3) and the human embryonic renal cell line (293T) cells were purchased from the Chinese Academy of Sciences Cell Bank and Cellbio (CAS, China). The cells were grown in basic RPMI-1640 medium (Gibco, USA) or DMEM (Gibco, USA) supplemented with $10 \%$ fetal bovine serum. All cells were maintained in a humidified $5 \% \mathrm{CO}_{2}$ atmosphere at $37^{\circ} \mathrm{C}$. 


\section{Transcriptome sequencing for breast specimens}

Transcriptome sequencing of 15 breast cancer tissues, 15 adjacent normal tissues and three breast tissues were obtained from HMUCC. A total of $3 \mu \mathrm{g}$ of RNA per sample was applied for RNA sample preparation. Ribosomal RNA was first removed using the Ribo-Zero ${ }^{\mathrm{TM}}$ Gold kit (Epicentre, Wisconsin, USA). Next, sequencing libraries were prepared according to the manufacturer's protocol, and the libraries were then sequenced on an Illumina HiSeq 2500 platform to generate 100-bp paired-end reads. Raw sequencing and processed RNASeq data have been deposited into the NCBI GEO database under accession number GSE71651 (https://www.ncbi.nlm.nih.gov/geo/query/acc.cgi?acc=GSE71651).

\section{BGISEQ-500 RNA-sequencing, ChIP-sequencing and small RNA-sequencing}

Total RNA was isolated from the cancer cells using the Promega isolation kit (Promega) according to the manufacturer's instructions. Raw sequencing and processed RNA-seq data were deposited in the NCBI GEO database under the accession number GSE124961

(https://www.ncbi.nlm.nih.gov/geo/query/acc.cgi?acc=GSE124961). For BGISEQ-500 ChIP-sequencing, six steps used for BGISEQ-500 ChIP-sequencing included sample detection, end repair and adaptor ligation, PCR, circularization, library quality control, and sequencing. Raw sequencing and processed CHIP-seq data were deposited in the NCBI GEO database under the accession number GSE124836 (https://www.ncbi.nlm.nih.gov/geo/query/acc.cgi?acc=GSE124836). For BGISEQ-500 small RNAsequencing, total RNA was isolated from the cancer cells using the Promega isolation kit (Promega) according to the manufacturer's instructions. Raw sequencing and processed small RNA-seq data were deposited in the NCBI GEO database under the accession number GSE124961 (https://www.ncbi.nlm.nih.gov/geo/query/acc.cgi?acc =GSE124961).

\section{Public data access}

Genome-wide expression profiles of LINC00036 and EGFR and clinical pathology information were downloaded from TCGA (https://tcga-data.nci.nih.gov/) and CCLE (http://www.broadinstitute.org/ ccle). All transcripts were normalized using $\log _{2}$ transformation. The expression of LINC00036 or EGFR was dichotomized by median expression as the cut-off to define high values (at or above the median) versus low values (below the median). An enrichment peak of c-MYC in the LINC00036 promoter was obtained from the ChIP-seq data of MCF-7 cells in the ENCODE portal (http://www.encodeproject.org). The putative c-MYC-binding site in the LINC00036 promoter was inspected using the JASPAR database

(jaspar.genereg.net/). Putative TF-binding site in the transcriptional start of LINC00036 locus are listed in Table S1. The predicted microRNAs binding to the 3'UTR of EGFR TargetScan and RNA22 are listed in Table S2. Venn diagrams were drawn using the VENNY 2.1 online software (https://bioinfogp.cnb.csic.es/tools/venny/). 


\section{Transient transfections, lentiviral transduction, and plasmid transfection}

For transfection, cells were transfected with $40 \mathrm{nM}$ siRNA targeting LINC00036, c-MYC, and PPP1R150, or a control siRNA using Lipofectamine ${ }^{T M} 2000$ (Invitrogen, \#11668019) or Lipofectamine ${ }^{T M} 3000$ (Invitrogen, \#L3000015) according to the manufacturer's instructions. Total RNA was isolated after $48 \mathrm{~h}$ for real-time PCR analysis. For transduction, lentiviruses were used to infect $5 \times 10^{5}$ cells in a 6 -well plate with 4-6 $\mu \mathrm{g} / \mathrm{ml}$ polybrene (Catalog number, 107689; Sigma). The infected cells were then subjected to selection with $1 \mu \mathrm{g} / \mathrm{ml}$ puromycin (Catalog Number 540411; Calbiochem, USA). Stable knockdown cell lines were identified using qRT-PCR. For plasmid transfection, cells were transfected with plasmid using Lipofectamine $^{\mathrm{TM}} 2000$ or Lipofectamine ${ }^{\mathrm{TM}} 3000$ according to manufacturer's recommendations. The siRNA/shRNA sequences are listed in Additional file 1: Table S1.

\section{Cell proliferation assay}

The cell counting kit-8 (Dojindo, Kumamoto, Japan) was used to assess cell growth. Approximately $2 \times$ $10^{3}$ cells per well were seeded in 96-well plates in a final volume of $100 \mu \mathrm{L}$ and then transfected with siLINC00036-1 or si-negative control (NC). The absorbance was detected $0,12,24,48$, and $72 \mathrm{~h}$ after gene transfection. CCK-8 solution $(10 \mu \mathrm{l})$ was added to each well and incubated for $2 \mathrm{~h}$ at $37^{\circ} \mathrm{C}$. The absorbance levels were measured at a wavelength of $450 \mathrm{~nm}$. All experiments were performed in triplicate. To calculate the half inhibitory concentration (IC50), data were fitted in GraphPad Prism 7.0, and the dose-response curve was plotted using the equation log (inhibitor) vs. response- variable slope. The IC50 was obtained using the following formula: Y=Bottom + (TopBottom) $/\left(1+10^{\wedge}((\right.$ Log IC $50-X)$ *HillSlope)).

\section{Cell apoptosis assay using flow cytometry}

The transfected cells were seeded in 6 -well plates $\left(5 \times 10^{5}\right.$ cells $/$ well $)$ and treated with $1.0 \mathrm{mg} / \mathrm{l}$ adriamycin. The cells were then digested with trypsin (Gibco® trypsin-EDTA, Thermo Fisher Scientific), washed thrice with PBS, suspended in $500 \mu$ binding buffer, and then incubated withFITC Annexin V Apoptosis Detection Kit I (BD Biosciences, 556547) according to the manufacturer's protocol. The stained cells were detected using the BD FACS Aria II flow cytometer (BD Biosciences, Hercules, CA, USA).

\section{Transwell migration/invasion assays}


Transwell migration assays (Corning ${ }^{\circledR}$ BioCoat ${ }^{\mathrm{TM}}$ control insert-No ECM, 8 micron pore size) and invasion assays (Corning BioCoat ${ }^{\mathrm{TM}}$ Matrigel invasion chamber) were performed according to the manufacturer's instructions. Cells which had migrated/invaded to the bottom of the insert were fixed with $70 \%$ ethanol and stained with $0.5 \%$ crystal violet. The migrated/invaded cells were photographed under an inverted microscope, quantified using ImageJ, and represented as percentage of total area.

\section{Western blot analysis}

Briefly, the protein concentrations were determined using a bicinchoninic acid protein assay kit and bovine serum albumin as the standard. Equal amounts of protein were fractionated via sodium dodecyl sulfate-polyacrylamide gel electrophoresis (SDS-PAGE) and transferred to polyvinylidene fluoride (PVDF) membrane (Millipore, Bedford, MA). The membranes were blocked for $2 \mathrm{~h}$ using $5 \%$ non-fat milk in Trisbuffered saline with Tween (TBST), and then probed overnight at $4{ }^{\circ} \mathrm{C}$. Following incubation with the primary antibodies, the membranes were incubated with secondary antibody (1: 8000 dilution, Alexa Fluor ${ }^{\circledR} 700$ goat anti-mouse $\lg G(\mathrm{H}+\mathrm{L})$ or Alexa Fluor ${ }^{\circledR} 800$ goat anti-rabbit $\lg G(\mathrm{H}+\mathrm{L})$; Invitrogen) in PBS at room temperature for $1 \mathrm{~h}$. The protein bands were imaged using the Odyssey infrared imaging system (LI-COR Biosciences, Lincoln, NE, USA) and quantified using the Odyssey v1.2 software (LI-COR Biosciences, Lincoln, NE, USA) by measuring the band intensity (area $\times \mathrm{OD}$ ) in each group. The band intensities were normalized to that of GAPDH or tubulin as the internal control. The western blot experiments were repeated thrice unless otherwise stated. The antibodies used are listed in Additional file 1: Table S2.

\section{Immunohistochemistry}

Paraffin-embedded tissue sections from patients with breast cancer were deparaffinized in xylene, rehydrated in a graded series of ethanol solutions, and then incubated for $20 \mathrm{~min}$ in $3 \% \mathrm{H}_{2} \mathrm{O}_{2}$ to block the endogenous peroxidase activity. Next, the sections were heated in target retrieval solution (Dako) for 15 min in a microwave oven (Oriental Rotor) to retrieve the antigen. Non-specific binding was blocked via incubation with $10 \%$ goat serum for $2 \mathrm{~h}$. The slides were then incubated overnight at $4{ }^{\circ} \mathrm{C}$ with the antiEGFR primary antibody. An appropriate secondary antibody was added and incubated for $20 \mathrm{~min}$ at 37 ${ }^{\circ} \mathrm{C}$, and binding was visualized with 3,39-diaminobenzidine tetrahydrochloride (DAB). After each treatment, the slides were washed thrice with TBST for $5 \mathrm{~min}$. Tissues were fixed in $4 \%$ neutral paraformaldehyde for at least $48 \mathrm{~h}$ and then embedded in paraffin. For safety evaluation of the nanocomplex, consecutive paraffin wax-embedded tissue sections of vital organs (4-5 $\mu \mathrm{m})$ were dewaxed, rehydrated, and stained with hematoxylin \& eosin (H\&E). The antibodies used are listed in Additional file 1: Table S1. 


\section{Quantitative real-time PCR}

Total RNA samples from cells and tissue samples were isolated using the Trizol reagent (Invitrogen, Carlsbad CA, USA) according to manufacturer's protocols. Total RNA (0.5 $\mu \mathrm{g})$ was then reverse transcribed using the High-Capacity cDNA reverse transcription kit (Applied Biosystems, MA, USA). The SYBR Green PCR master mix (Applied Biosystems, USA) was used to quantify the RNA levels, and GAPDH or U6 was used as an internal control. The qRT-PCR was performed on a 7500 FAST real-time PCR system (Applied Biosystems, USA). The primers used are listed in Additional file 1: Table S3, 4. LINC00036 and mRNA levels were normalized to those of GAPDH, while microRNAs levels were normalized to those of small nuclear $U 6$. The $2^{-\Delta \Delta C}$ t method was used to calculate relative expression levels.

\section{Terminal deoxynucleotidyl transferase dUTP nick end labeling assay}

Apoptosis-induced DNA fragmentation was determined using the terminal deoxynucleotidyl transferase dUTP nick end labeling (TUNEL) assay. The cells or tissues under different experimental conditions were fixed with $4 \%(\mathrm{w} / \mathrm{v})$ paraformaldehyde and processed using a commercial kit (Roche) in accordance with the manufacturer's instructions. Tumor samples were fixed for $30 \mathrm{~min}$, rinsed with PBS, blocked for 10 min using a solution of $96 \%$ methanol mixed with $4 \% \mathrm{H}_{2} \mathrm{O}_{2}$ at room temperature, and permeabilized with $0.2 \%$ Triton $\mathrm{X}-100$ in PBS for 5 min at $4{ }^{\circ} \mathrm{C}$. The TUNEL staining was performed using the in situ cell death detection kit (Roche) and the nuclei were stained with DAPI (Beyotime, China) for $10 \mathrm{~min}$. The number of TUNEL-positive cells and the total number of cells were captured using a fluorescence microscope (IX71 + DP72, Olympus, Japan) and apoptosis was determined using the ImagePro Plus software.

\section{Isolation of nuclear/cytoplasmic fractions}

Nuclear/cytoplasmic fractions were isolated using the NE-PERTM nuclear and cytoplasmic extraction reagents (Catalog number 78835; Thermo Fisher) according to the manufacturer's protocol. Cytoplasmic and nuclear fractions were divided for RNA extraction. The GAPDH and U1 enzymes were used as qRTPCR markers for the cytoplasmic and nuclear RNAs, respectively.

\section{RNA fluorescent in situ hybridization (RNA-FISH)}

ViewRNA®Probe (Catalogue Number VA1-3016120, Santa Clara) was purchased to perform Fluorescence in situ hybridization (FISH) assay according to the manufacturer's protocol. LINC00036 hybridization was carried out in a moist chamber. After digestion with a working protease solution, slides were incubated with RNase III (AM2290, Life Technologies, USA) or RNase A (AM2272, Life Technologies) for $2 \mathrm{~h}$. Standard immunofluorescence and imaging were performed by confocal microscopy. 


\section{Actinomycin D assay}

Cells were treated with $5 \mu \mathrm{M}$ (final concentration) actinomycin D (Catalog number A1410; Sigma; dissolved in $100 \%$ ethanol). Actinomycin $\mathrm{D}$ was added to cells $0,1,2,4,6,12$, or $24 \mathrm{~h}$ prior to RNA extraction with the TRIzol reagent. Subsequently, qRT-PCR was used to analyze the changes in the RNA levels.

\section{RNA immunoprecipitation (RIP) and RNA pull down assays}

RIP was performed using a Magna RNA-binding protein immunoprecipitation kit (Millipore, Bedford, MA) according to the manufacturer's instructions. Briefly, cell lysates were incubated with RIP buffer containing magnetic beads conjugated with negative control normal mouse IgG or human antiPPP1R150 antibody. The samples were then incubated with proteinase $K$ to isolate the immunoprecipitated RNA. Finally, purified RNAs were extracted and analyzed using real-time PCR to confirm the presence of the binding targets. RNA pull-down was performed using a magnetic RNA-protein pull-down kit (Pierce Biotechnology, USA) in accordance with the manufacturer's instructions.

\section{Chromatin immunoprecipitation}

Chromatin immunoprecipitation (ChIP) was performed using the EZ ChIP kit (Millipore, Bedford, MA, USA) according to the manufacturer's protocol. In total, $1 \times 10^{6}$ cells were fixed in $1 \%$ formaldehyde at room temperature for $10 \mathrm{~min}$, and the nuclei were isolated with nuclear lysis buffer supplemented with a protease inhibitor. The chromatin DNA was sonicated and sheared to 100-200 bp fragments. The sheared chromatin was immunoprecipitated at $4{ }^{\circ} \mathrm{C}$ overnight using an anti-c-MYC antibody (Cell Signaling Technology). Normal mouse IgG was used as the negative control and an anti-RNA pol II antibody (Millipore) was used as the positive control.

\section{Luciferase reporter assay}

Luciferase reporter vector-harboring lentiviral particles were constructed with the wild type or mutant $3 \dot{c}$ UTR of EGFR or LINC00036 and packaged by Shanghai GeneChem, Co., Ltd. The H293T cells were transfected with wild type or mutant reporter plasmid vector using Lipofectamine 2000 and were then cotransfected with microRNA mimics or the negative control. After $48 \mathrm{~h}$, luciferase assays were performed using the dual luciferase assay system (Promega, catalogue E1910). Firefly luciferase activity was normalized to the Renilla luciferase activity for each sample. 


\section{In vivo xenograft model}

All experimental procedures involving animals were performed in accordance with the animal protocols approved by the Animal Care and Use Committee of Harbin Medical University. Briefly, 4-5-week-old female BALB/c nude mice were obtained from the Shanghai Laboratory Animal Center (CAS), housed in a pathogen-free and temperature-controlled environment. Stably transfected cancer cells $\left(1 \times 10^{7}\right.$ UACC812 cells, $5 \times 10^{6}$ SGC-7901 cells and $5 \times 10^{6} \mathrm{NCI}-\mathrm{H} 1975$ cells $囚$ in $100 \mu$ l PBS together with anequal volume of Matrigel basement membrane matrixwere injected subcutaneously into the right abdomento establish breast cancer xenograft model, lung cancer xenograft model and gastric cancer xenograft model respectively. One week after the tumor cell inoculation, the mice were treated with gefitinib at 20 $\mathrm{mg} / \mathrm{kg} /$ day by asingle intraperitoneal injection (i.p). Tumor volumes were measured every 4 days after being apparently observed and calculated with the following formula: Volume $=\left(\right.$ length $\times$ width $\left.^{2}\right) / 2$. After 30 days, all mice were sacrificed under anesthesia.

\section{LINC00036 targeting nanoparticles preparation and biodistribution}

The LINC00036 targeting ASO was designed and synthesized by Integrated DNA Technologies (Skokie, Illinois, USA). The ASO and DOTAP liposomal transfection reagent were dissolved in methylene dichloride and allowed to stand for $20 \mathrm{~min}$ at room temperature according previous studies [43, 44]. Next, the mixture was rehydrated in normal saline for future use. The mice of the control group were administrated with $0.9 \%$ normal saline via tail vein injection. The treatment groups were administrated with $20 \mu$ ASO or $20 \mu \mathrm{l}$ LINC00036 targeting nanoparticles (TTN) via tail vein injection (t.v.i). Three groups (four mice per

group) of female BALB/c nude mice were used to evaluate the biodistribution of the TTN in vivo. Cyanine 5.5 was used to label TTN and ASO. The mice of the control group were administrated $200 \mu 10.9 \%$ normal saline and the treatment groups were administrated $150 \mu$ cyanine $5.5 /$ ASO or $150 \mu$ l cyanine $5.5 /$ TTN via tail vein injection at time intervals of 1, 3, and $24 \mathrm{~h}$. Imaging of cyanine 5.5-labeled ASO DNA oligo was monitored over time by detecting near infrared (NIR) fluorescence in the whole animal according to the instructions of the IVIS Lumina Imaging System 100 series (Xenogen, Alameda, CA, USA).

\section{Statistical analysis}

All statistical analyses were performed using the R 4.1.2 (R Foundation for Statistical Computing, Vienna, Austria) software program or GraphPad Prism 7 software (San Diego, CA). All immunohistochemistry, immunoprecipitation, western blot, and in vitro assays were conducted for more than three times to ensure reproducibility. The number of mice in each group has been indicated for specific in vivo experiments. Correlations between the LINC00036 and EGFR expression were assessed using Pearson 
correlation coefficients. Unpaired Student's t-tests were used to detect significant differences among tumors or between tumor and normal samples. The OS was calculated as the time from surgery until the occurrence of death. The DSS indicated the duration of disease-specific survival. Survival curves were constructed using the Kaplan-Meier method and were compared between subgroups with the log-rank test. All $P$ values were two-tailed, and $P<0.05$ was considered statistically significant. All data are presented as the mean \pm standard deviation (SD) from at least three independent replicates.

\section{Results}

\section{LINC00036 is overexpressed and is a c-MYC inducible IncRNA in human cancer}

To identify novel onco-IncRNAs associated in breast cancer『we determined the changes in IncRNAs expression by comparing the expression profile in 15 breast cancer tissues and paired adjacent normal tissues, and 3 normal breast tissues using high-throughput transcriptome sequencing. In the present study, we mainly focused on the upregulated IncRNAs given that these IncRNAs might serve as therapeutic targets or biomarkers. Based on the high-throughput transcriptome sequencing, LINC00036, which was one of the prominently upregulated IncRNAs in breast cancer tissues, was chosen for further evaluation (Fig. 1a). Next, 185 breast cancer samples, 36 lung cancer samples, 32 gastric cancer samples and 21 glioma samples from HMUCC were applied to validate the result. According to the qRT-PCR confirmation results, LINC00036 was found to be upregulated in these human cancers (Fig.1b-e). Moreover, elevated expression of LINC00036 was also validated in various types of cancer (including BLCA, BRCA, CESC, CHOL, ESCA, LUSC, PCPG, SKCM and THCA) based on The Cancer Genome Atlas (TCGA) database (Fig. 1f).

Next, the possible transcription factor-binding sites in the LINC00036 promoter were detected and putative c-MYC-binding site was identified (Fig. 1g and Additional file 1: Table S5). Recent studies have shown that aberrant c-MYC activation drives key gene networks contributing to the pathogenesis of many human cancers, and an increasing number of IncRNAs have been found to be involved in C-MYCmediated signaling [45-47]. Gene Expression Omnibus (GEO) and TCGA were used to investigate relationship between c-MYC and LINC00036 in the human cancer and the knockdown of c-MYC decreased the expression of LINC00036 in GSE5823 (Additional file 2: Fig. S1A). Moreover, c-MYC expression was positively correlated with LINC00036 expression in 9,670 patients in 33 types of cancer from TCGA data (Fig. 1h and Additional file 2: Fig.S1B-D). Notably, to validate the result, c-MYC knockdown significantly reduced LINC00036 expression in five cancer cell types (Fig. 1i), whereas c-MYC overexpression observably upregulated LINC00036 in these cancer cells (Fig. 1j). ChIP-seq was performed to avoid false positive results and correctly interpret the mechanism of LINC00036 regulation. The results revealed significant enrichment of C-MYC peaks in the LINC00036 promoter (chr19-5571145-5571442) in UACC-812 cells (Fig. 1k, upper panel). Furthermore, obvious enrichment of c-MYC peaks was observed in the promoter region of LINC00036 in MCF-7 cells in the ENCODE database (Fig. 1k, bottom panel). This observation was verified using ChIP-PCR, which is in agreement with the results of ChIP-seq (Fig.1I). 


\section{LINC00036 acts as an oncogenic IncRNA in human cancer}

To address biological function of LINC00036 in human cancer, the UACC-812 and MDA-MB-453 cells were transduced with lentiviru encoding shortin terfering RNAs (shRNA) for targeting LINC00036 expression (Additional file 2: Fig. S2A). The CCK-8 assay revealed that knockdown of LINC00036 significantly impaired breast cancer cell proliferation in UACC-812 and MDA-MB-453 cells (Additional file 2: Fig.S2B). Moreover, the measurement of apoptosis using flow cytometry analysis and western blot analysis revealed that LINC00036 knockdown increased the apoptosis, in contrast to the Sh-NC group (Additional file 2: Fig.S2C-E). Furthermore, LINC00036 knockdown also decreased the migration and invasion motility in UACC-812 and MDA-MB-453 cells (Additional file 2: Fig.S2F-I). All the above findings uncovered a novel function of knockdown LINC00036 inhibits cell growth and migration/invasion and accelerates cell apoptosis in vitro. Short hairpin RNA (shRNA) targeting (sh-) LINC00036-1 was selected for future experiments based on the qRT-PCR results. Next, we silenced LINC00036 with shRNA in different cell lines (incuding lung cancer cell line NCI-H1975, gastric cancer cell line SGC-7901, kidney cancer cell line 786-0, glioma cell line U251, liver cancer cell line Hep3B and ovarian cancer cell line SKOV-3) (Additional file 2: Fig. S2J). Of note, the CCK-8 assay revealed that LINC00036 knockdown also significantly inhibited these cells proliferation (Fig. 2a).

To further verify the result of LINC00036 on tumor growth in vivo, we developed mouse subcutaneous xenografts model using UACC-812, NCl-H1975, and SGC-7901 cell transfected with lentivirus. The LINC00036 knockdown significantly retarded the growth of breast cancer, lung cancer, and gastric cancer xenografts (Fig. 2b-g). Next, the TUNEL assay indicated an increase in the number of apoptotic cells in the LINC00036 knockdown group than that of the Sh-NC group (Fig. 2h, i). Furthermore, compared to that in the Sh-NC groups, tumors in the LINC00036 knockdown group showed increased Bax expression and reduced $\mathrm{Bcl}-2$ expression (Fig. 2j). Collectively, these in vivo experiment results were consistent with in vitro data that not only indicative of the oncogenic activity of LINC00036, but also suggested that it can be used as a potential therapeutic target for cancer treatment.

\section{LINC00036 is a promising novel therapeutic target}

A potent effective therapeutic model, ASO-targeting LINC00036 coupled with cationic lipid 1,2-dioleoyl-3 trimethylammonium-propane (DOTAP) [44, 48], which is hereafter named as LINC00036 targeting nanoparticle (TTN), was developed and applied in the following experiments. On an average, TTN were $\sim 90 \mathrm{~nm}$ in diameter (Fig. 3a) and showed higher tumor accumulation than ASO alone at different time points. It was retained for at least $24 \mathrm{~h}$ in xenograft models of breast cancer, gastric cancer, and lung cancer (Fig. 3b). Compared to that in the ASO group and blank control group, treatment with TTN markedly reduced tumor growth (Fig. 3c-e). The TUNEL assay indicated the presence of more apoptotic cells in the TTN treatment group than in the other groups (Fig. $3 \mathrm{f}$ and Additional file 2: Fig. S3A). 
Furthermore, in accordance with the above results, tumor samples in the TTN treatment group showed increased Bax expression and decreased Bcl-2 expression (Fig. 3g).

In addition, different organs were harvested to investigate the toxicity effects of TTN treatment. No observable toxicity was observed in these organs in TTN treatment, although mild apoptosis and necrosis were observed in the TUNEL assay and H\&E staining (Additional file 2: Fig.S3B-D). Venous blood samples were also collected to investigate the biochemical indexes after TTN treatment and the result showed that the TTN treatment negligibly affected the function of the liver, kidney, and heart (Additional file 2: Fig. S3E).Thus, LINC00036 is a promising novel therapeutic target and targeting of LINC00036, especially coupled with a potent DOTAP, which may represent a novel next generation anti-cancer therapeutic strategy.

\section{LINC00036 ignites the PI3K/AKT signaling pathway and MAPK signaling pathway through up-regulation EGFR expression}

To determine the function of LINC00036, RNA-seq was performed using UACC-812 cells transfected with three siRNAs individually targeting LINC00036. Results revealed that EGFR transcription decreased significantly in the LINC00036 knockdown group compared to that in the scramble group (Fig. 4a). EGFR has been reported to promote progression via the AKT and MAPK signaling pathway in human cancer [49-51]. EGFR expression was significantly inhibited by LINC00036 knockdown both at mRNA and protein levels in UACC-812, NCI-H1975 and SGC-7901 (Fig. 4b). Furthermore, LINC00036 knockdown decreased pEGFR, p-AKT, p-MEK and p-ERK expression in the above three cancer cell lines and xenografts (Fig. 4c, d). Positive correlation expression between LINC00036 and EGFR was observed in 50 samples from breast cancer patients in HMUCC (Fig. 4e). Furthermore, the relationship between LINC00036 and EGFR protein expression was next analyzed in 154 breast cancer tissue samples from HMUCC using immunohistochemical staining. Results demonstrated that EGFR was abundant in the tissues with LINC00036-high expression group and vice versa (Fig. 4f, g). Next, we integrated multi-dimensional data from TCGA and CCLE projects to characterize the relationship between the EGFR and LINC00036 transcription landscapes. As expected, LINC00036 expression correlated positively with EGFR mRNA levels in 9 common types of cancer tissues including breast cancer in TCGA cohort (Fig. 4h and Additional file 2: Fig.S4A-I). In addition, a similar result was obtained in 602 cancer cell lines across 33 cancer types from the CCLE project, which was in agreement with TCGA data (Fig. 4i).

\section{LINC00036 stabilizes EGFR expression via RNA-protein interaction and acts as a competing endogenous RNA (ceRNA)}

LncRNAs can regulate gene expression via transcriptional interference in cis or in trans manner. The increased EGFR mRNA levels may be result of increased transcription or enhanced mRNA stability. To 
further investigate how LINC00036 contributes to EGFR expression, we first detected LINC00036 subcellular localization in eight diffrent cancer cells by nucleocytoplasmic fractionation PCR analyses. The results showed that LINC00036 was located both in nuclear and cytoplasm, while predominant within the cytoplasm of cancer cells, which was further verified by single-molecule RNA fluorescence in situ hybridization (FISH) (Additional file 2: Fig.S5A, B), and suggesting that LINC00036 might be involved in post-transcriptional regulation and RNA interactions $[52,53]$. The half-life of the EGFR mRNA was shorter in LINC00036 knockdown cells than in the control cells, as observed after treatment of the breast cancer cells with actinomycin D (Fig. 5a, b). A report indicates that PPP1R150 increases the stability of differentiation-related mRNAs in somatic tissues [54, 55].Therefore, siRNA targeting PPP1R150 was designed to investigate the role of PPP1R150 in EGFR mRNA stability (Additional file 2: Fig.S5C). Results showed that the half-life of the EGFR mRNA was shorter in the PPP1R150 knockdown group than that in the control (Fig. 5c). Furthermore, RNA binding protein immunoprecipitation assays showed significant LINC00036 and EGFR enrichment in the PPP1R150 group compared to that in the IgG group (Fig. $5 d$, e). The RNA pull-down assays showed significant enrichment of PPP1R150 in the LINC00036 group than in the control group (Fig. 5f). Therefore, LINC00036 enhanced EGFR mRNA stability via the LINC00036-PPP1R150 interaction.

In addition to the regulation of mRNA stability, IncRNAs located within the cytoplasm affect miRNAmediated gene regulation by acting as ceRNA or natural miRNA sponges [54]. To investigate whether LINC00036 functions as a ceRNA for regulating EGFR mRNA expression, small RNA sequencing was performed and 138 potential microRNAs candidates that potentially bind to the LINC00036 transcript were identified (Fig. 5g). Furthermore, public databases, including TargetScan and RNA22, were used to predict the microRNAs binding to the 3द UTR of EGFR (Additional file 1: Table S6). Next, overlapping microRNAs were analyzed among the upregulated microRNAs in the LINC00036 knockdown sequencing data and TargetScan and RNA22 databases. Finally, 74 candidate microRNAs were obtained though this filtration process (Additional file 2: Fig.S5D). Quantitative reverse transcription (qRT)-PCR was performed to validate these 74 candidate microRNAs, and 12 microRNAs were finally identified to be upregulated significantly after LINC00036 knockdown (Fig. 5h). Furthermore, inhibitors for these 12 microRNAs were designed to examine LINC00036 expression. As expected, increase in LINC00036 expression was observed after treatment with these microRNA inhibitors (Additional file 2: Fig.S5E, F). Subsequently, EGFR mRNA and protein levels significantly decreased or increased when microRNA mimics or inhibitors, respectively, were applied (Additional file 2: Fig.S5G, H and Fig. 5i, j). The luciferase reporter assays showed that the wild-type 3C UTR of EGFR or LINC00036 was regulated by miR-125b-2-3p and miR-424$5 p$ and that this effect was abolished by mutating the binding sites (Fig. 6a-d). Our results indicated that EGFR and LINC00036 were bona fide direct targets of miR-125b-2-3p and miR-424-5p. In light of the above findings, we hypothesized that c-MYC/LINC00036/miR-125b-2-3p/miR-424-5p/EGFR axis might play vital role in the tumorigenesis. The UACC-812 and MDA-MB-453 cells were transfected with different vectors/inhibitor/sh-LINC00036 and LINC00036 knockdown decreased cell proliferation significantly, whereas miR-125b-2-3p and miR-424-5p inhibitors markedly rescued theses phenotypes (Fig. 6e, f). Meanwhile, we obtained a similar result in lung cancer cell line NCI-H1975 and stomach cancer cell line 
SGC-7901 with different assignment (Additional file 2: Fig.S6A, B). Application of miR-125b-2-3p and miR424-5p inhibitors attenuated the suppression of EGFR, p-EGFR, p-ATK, p-MAPK, p-ERK and c-MYC in cancer cells with LINC00036 knockdown and vice versa (Fig. 6g). These results demonstrated that cMYC/LINC00036/miR-125b-2-3p/miR-424-5p/EGFR axis promotes the tumorigenesis in human cancer.

\section{LINC00036 knockdown enhances human cancer cell sensitivity to gefitinib in vitro and in vivo}

To determine the role of LINC00036 on gefitinib sensitivity, multiple types of cancer cells were exposed with different concentrations of gefitinib for $48 \mathrm{~h}$. CCK8 assays showed that knockdown of LINC00036 decreased the gefitinib IC50 value compared with that of the control cells, respectively (Additional file 2: Fig.S7A-H). To further confirm the role of LINC00036 in gefitinib sensitivity, we examined the effects of LINC00036 on cell proliferation with sh-IC50 concentration gefitinib treatment. As shown in Fig.7a, CCK8 proliferation assays showed that LINC00036 knockdown significantly inhibited cell proliferation compared with the control cells with sh-IC50 concentration gefitinib treatment. Moreover, LINC00036 knockdown decreased phosphorylation levels of EGFR significantly in human cancer cells (Fig. 7b).

In addition, we attempted to develop co-targeting therapies by combining gefitinib with TTN and investigate the synergistic anti-tumor effects. As expected, the combination of gefitinib with TTN improved tumor suppression (Fig. 7c, d) and increased the cancer cell apoptosis compared to that with gefitinib or TTN alone (Fig. 7e and Additional file 2: Fig.S7I). Furthermore, western blot analysis showed that combination of gefitinib with TTN inhibited the cascade of EGFR and its downstream signaling pathway (Fig. 7f). Moreover, lung adenocarcinoma patients with high expression of LINC00036 showed less gefitinib sensitivity than patients with low expression of LINC00036 in GSE11969 dataset (Additional file 2: Fig. 7J).

\section{High LINC00036 expression is associated with poor prognosis}

The association of LINC00036 expression with patient survival was analyzed in 185 patients with breast cancer in the HMUCC cohort. Patients harboring tumors with upregulated LINC00036 expression were associated with potential poor overall survival (Fig. 8a). Owning to the limit of patient follow-up span and the sample number in the HMUCC cohort, 33 cancer types in 14,586 cancer patients in TCGA were used to compensate for the shortage of HMUCC data and to evaluate whether the upregulated C-MYC-LINC00036EGFR axis was robustly associated with the poor survival of patients. As expected, patients in LINC00036 high/EGFR-high group was found to be consistently associated with poor overall survival (Fig. 8b). Furthermore, patients with LINC00036-high $/ \mathrm{MYC}^{\text {-high }}$ expression also significantly correlated with poor disease special survival (Fig. 8c). Notably, patients with LINC00036-high $/$ EGFR $^{\text {-high }} /$ c-MYC $^{\text {-high }}$ expression showed worse overall survival and disease special survival (Fig. 8d, e). Overall, these data indicated that the c-MYC-LINC00036-EGFR axis correlated with poor prognosis of cancer patients (Fig. 8f, left). 


\section{Discussion}

Recently, aberrant expression of onco-IncRNAs has been reported in various cancers [2,56,57], and oncoIncRNAs have been shown to play important roles in tumor progression $[58,59]$. Identification of novel onco-IncRNAs, characterization of their function and regulatory mechanisms, and development of novel IncRNA-based targeted strategies for combating cancer appear promising and may introduce a new paradigm in cancer research. In this study, we identified that LINC00036, a c-MYC-activated onco-IncRNA, promotes cancer progression and decreases the sensitivity of gefitinib in human cancer via RNA-protein and RNA-RNA interactions. Here, we attempted to identify a novel onco-IncRNA and develop a feasible onco-IncRNA-targeted therapeutic strategy against cancer.

Base on transcriptome RNA sequencing and the human cancer samples analysis from HMUCC, we demonstrated for the first time that LINC00036 is overexpressed in human cancer (Fig.1) and the oncoprotein C-MYC was the transcriptional regulator of LINC00036, as it can bind to its promoter region (chr 19-5571145-5571442)(Fig.1). As a master transcriptional factor, C-MYC regulates the expression of approximately $10-15 \%$ genes in the genome [60], including various protein-coding genes [61], as well as various IncRNAs $[62,63]$. Here, LINC00036 was shown to connect the c-MYC and EGFR axis, which correlated with poor survival. Our study uncovered a distinctive facet of tumorigenesis, which involves the novel positive feedback loop of the c-MYC-LINC00036-EGFR signaling pathway. It also provides a broad framework for understanding the regulation of EGFR tyrosine kinase signaling by IncRNAs, suggesting that LINC00036 may be a new therapeutic target in pharmacological strategies for human cancer. Thus, this is the first study reporting the existence and function of the IncRNA-EGFR axis.

Recent clinical trials have demonstrated that novel therapeutic regimens targeting EGFR, including monoclonal antibodies, tyrosine kinase inhibitors, phosphatidylinositol 3-kinase inhibitors, and antisense gene therapy, can effectively treat diverse tumors, such as head and neck [64-66], bladder [67], lung [32], ovarian [68,69], and cervical cancers [70]. However, the therapeutic efficiency of single-agent treatment is variable [71]. Co-targeting therapies as an important step toward improving the clinical outcome of patients with advanced cancer should be encouraged [72-74]. Effective to co-targeting requires the identification of other critical modulators of the oncogenic cascade that drives disease progression and the development of strategies that can target EGFR pathways. In this study, co-targeting of LINC00036 and EGFR via LINC00036 knockdown and gefitinib synergistic therapy significantly enhanced antitumor efficacy and gefitinib-sensitivity (Fig.8), indicating that LINC00036 alone or in combination with EGFR inhibitors can overcome resistance to EGFR-targeted therapies.

Generally, IncRNAs interact with chromatin DNA, mRNA, or protein, thereby regulating chromatin accessibility, mRNA stability, and protein activity or stability in cis or in trans manner [74-77]. Unlike the classical theory, the identification of LINC00036's RNA-RNA and RNA-protein-mediated dual regulation in this study sheds light on the IncRNA-mediated complex regulation of its target genes. We showed that LINC00036 acts as an EGFR mRNA stabilizer, thereby inducing the cascade of the downstream AKT and MAPK signaling pathways, which in turn directly promote tumorigenesis (Fig.4). Mechanistically, 
LINC00036 upregulated EGFR expression by recruiting PPP1R150, promoting EGFR mRNA stability (Fig.5). In addition to the RNA-protein model, we have revealed another parallel ceRNA model. A systematic screening of microRNAs has been performed in this study to identify potential ceRNA candidates regulating LINC00036 and EGFR expression. Furthermore, our study suggests that LINC00036 functions as a ceRNA to upregulate EGFR expression by acting as a sponge for miR-125b-2-3p and miR424-5p (Fig.5 and Fig.6). Thus, this study fills in the theoretical gap in the IncRNA regulation model and provides comprehensive understanding regarding the above-mentioned dual regulatory network in human cancer.

LncRNAs can be targeted by multiple mechanisms with diverse modes of action. Of them, ASOs are attractive candidates for targeted therapeutic delivery. On one hand, post-transcriptional RNA degradation pathways can knock down pathogenic RNAs. This can be achieved using siRNAs that trigger a Dicer and Argonaute-dependent cleavage pathway. Alternatively, ASOs with chemical modifications can be used to target the RNA of interest for degradation via an RNAse H-dependent mechanism. On the other hand, one can also achieve loss of function via steric inhibition of RNA-protein interactions or prevention of secondary structure formation. RNA binding small molecules or ASOs can be used in this case. However, a drug delivery system with limited enhanced permeability and retention effect may result in poor therapeutic efficacy due to inefficient drug delivery to the tumor. To avoid this disadvantage, we have developed a more potent delivery system, namely, the ASO-targeting LINC00036 coupled with a potent DOTAP, to inhibit LINC00036 expression in mice xenograft models (Fig.3). However, LINC00036 was targeted using this delivery system in mice xenograft models, which is a limitation of this study, further investigations on large animal or primate models are desirable.

\section{Conclusions}

In summary, we have identified LINC00036, a c-MYC inducible onco-IncRNA, acts an oncogene in human cancer and decreases the sensitivity of gefitinib through positive feedback loop of the C-MYC-LINC00036EGFR axis (Fig.8, left pannel). Furthermore, the identification of RNA-RNA and RNA-protein dual regulation model sheds light on the IncRNA-mediated complex regulation of target genes. Finally, a new paradigm may be discovered, in which targeting of LINC00036, especially coupled with a potent DOTAP, represents a novel next generation anti-EGFR therapeutic strategy via improving the sensitivity of gefitinib (Fig.8, right pannel). All results obtained were based on large number of patient tissue samples, warranting further clinically actionable investigation, which would assist in custom-designing treatments for various cancers.

\section{Abbreviations}

ALP: Alkaline phosphatase; BLCA: Bladder Urothelial Carcinoma; BRCA: Breast cancer; BUN: Blood urea nitrogen; CCLE: Cancer Cell Line Encyclopedia; ceRNA: Competing endogenous RNA; CESC: Cervical squamous cell carcinoma and endocervical adenocarcinoma; ChIP: Chromatin immunoprecipitation; ChIP-seq: Chromatin immunoprecipitation-sequencing; COAD: Colon adenocarcinoma; CRE: Creatinine; 
DSS: Disease special survival; ESCA: Esophageal carcinoma; FISH: Fluorescence in situ hybridization; GGT: y-glutamyl transpeptadase; GPT: Glutamic pyruvic transaminase; HMUCC: Harbin Medical University Cancer Center cohort; HNSC: Head and Neck squamous cell carcinoma; LIHC: Liver cancer; LncRNA: Long non-coding RNA; LUSC: Lung squamous cell carcinoma; NL: Normal-like; OS: Overall survival; PAAD: Pancreatic adenocarcinoma; PCPG: Pheochromocytoma and Paraganglioma; PFI : Progression free interval; PRAD: Prostate adenocarcinoma; qRT-PCR: Quantitative real-time PCR; READ: Rectum adenocarcinoma; SKCM: Skin Cutaneous Melanoma; STAD: Stomach adenocarcinoma; TBIL: Total bilirubin; TCGA: The Cancer Genome Atlas; THCA: Thyroid carcinoma; THYM: Thymoma.

\section{Declarations}

\section{Acknowledgements}

Not applicable.

\section{Authors' contributuons}

Pa D and Xu SP designed the project. Wan L, Wang Q, Yin HZ, Qiao K, Zhang JF, Wu H and Shen MY performed the experiments. Wan $L$ and Wang Q processed the data. Pa D, Xu SP and Wan $L$ wrote the manuscript. Yin HZ, Li SW and Ning SP collected the human cancer samples and clinical information. All authors read and approved the final manuscript.

\section{Funding}

This work was supported by funding from the Project Nn10 of the Harbin Medical University Cancer Hospital (No.102017-02), the National Natural Science Foundation of China (No. 82072904, 81972706ه 81872149) and the Outstanding Youth Project of Heilongjiang Provincial Natural Science Foundation (No.YQ2019H027).

\section{Availability of data and materials}

Not applicable.

\section{Ethics approval and consent to participate}

Patient tissue samples were obtained with informed consent, under theprotocol approved by the ethical review board of the Harbin Medical University Cancer Hospital 
Consent for publication

Consent for publication is not applicable for this article

\section{Competing interests}

The authors declare that they have no competing interests

\section{References}

1. Tragante V, Moore J, Asselbergs F: The ENCODE project and perspectives on pathways. Genetic epidemiology 2014, 38:275-280.

2. Luo H, Zhu G, Xu J, Lai Q, Yan B, Guo Y, Fung T, Zeisig B, Cui Y, Zha J, et al: HOTTIP IncRNA Promotes Hematopoietic Stem Cell Self-Renewal Leading to AML-like Disease in Mice. Cancer cell 2019, 36:645-659.e648.

3. Wahlestedt C: Targeting long non-coding RNA to therapeutically upregulate gene expression. Nature reviews Drug discovery 2013, 12:433-446.

4. Schmitt A, Chang H: Long Noncoding RNAs in Cancer Pathways. Cancer cel/ 2016, 29:452-463.

5. Melo CPDS, Campos CB, Rodrigues JDO, Aguirre-Neto JC, Assumpo JG: Long non-coding RNAs: Biomarkers for acute leukaemia subtypes. British Journal of Haematology 2015, 173.

6. Klingenberg M, Matsuda A, Diederichs S, Patel T: Non-coding RNA in hepatocellular carcinoma: Mechanisms, biomarkers and therapeutic targets. Journal of hepatology 2017, 67:603-618.

7. Veltri W: Non-coding RNAs as biomarkers for metastatic prostate cancer. The Lancet Oncology 2014, 15:1412-1413.

8. Thomas R., Cech, Joan A., Steitz: The Noncoding RNA Revolution-Trashing Old Rules to Forge New Ones. Cell 2014.

9. Lennox KA, Behlke MA: Cellular localization of long non-coding RNAs affects silencing by RNAi more than by antisense oligonucleotides. Nuclc Acids Research 2016:863-877.

10. Wen, Shen, Xue-hai, Liang, Stanley, T., Crooke: Phosphorothioate oligonucleotides can displaceNEAT1RNA and form nuclear paraspeckle-like structures. Nucleic Acids Research 2014.

11. Genetic variants and cellular stressors associated with exfoliation syndrome modulate promoter activity of a IncRNA within the LOXL1 locus. Human Molecular Genetics 2015.

12. Shechner DM, Hacisuleyman E, Younger ST, Rinn JL: Multiplexable, locus-specific targeting of long RNAs with CRISPR-Display. Nature Methods 2015, 12:664-670.

13. Ling $\mathrm{H}$, Fabbri $\mathrm{M}$, Calin GA: MicroRNAs and other non-coding RNAs as targets for anticancer drug development. Nature Reviews Drug Discovery 2013, 12:847-865. 
14. Mercer T, Mattick J: Structure and function of long noncoding RNAs in epigenetic regulation. Nature structural \& molecular biology 2013, 20:300-307.

15. Sullenger BA, Nair S: From the RNA world to the clinic. ence 2016, 352:1417-1420.

16. Gutschner T, Hammerle M, Eissmann M, Hsu J, Kim Y, Hung G, Revenko A, Arun G, Stentrup M, Gross M: The Noncoding RNA MALAT1 Is a Critical Regulator of the Metastasis Phenotype of Lung Cancer Cells. Cancer Research 2012.

17. Arun G, Diermeier S, Akerman M, Chang KC, Spector DL: Differentiation of mammary tumors and reduction in metastasis upon Malat1 IncRNA loss. Genes \& Development 2016, 30:34.

18. Wheeler TM, Leger AJ, Pandey SK, Macleod AR, Nakamori M, Cheng SH, Wentworth BM, Bennett CF, Thornton CA: Targeting nuclear RNA for in vivo correction of myotonic dystrophy. Nature 2012, 488:111-115.

19. Kim J, Piao HL, Kim BJ, Yao F, Han Z, Wang Y, Xiao Z, Siverly AN, Lawhon SE, Ton BN: Long noncoding RNA MALAT1 suppresses breast cancer metastasis. Nature Genetics 2018, 50.

20. Lu Y, Zhao X, Liu Q, Li C, Graves-Deal R, Cao Z, Singh B, Franklin J, Wang J, Hu H, et al: IncRNA MIR100HG-derived miR-100 and miR-125b mediate cetuximab resistance via Wnt/ $\beta$-catenin signaling. Nature medicine 2017, 23:1331-1341.

21. Esposito R, Bosch N, Lanzós A, Polidori T, Pulido-Quetglas C, Johnson R: Hacking the Cancer Genome: Profiling Therapeutically Actionable Long Non-coding RNAs Using CRISPR-Cas9 Screening. Cancer cell 2019, 35:545-557.

22. Zhou C, Yi C, Yi Y, Qin W, Yan Y, Dong X, Zhang X, Huang Y, Zhang R, Wei J, et al: LncRNA PVT1 promotes gemcitabine resistance of pancreatic cancer via activating Wnt/ $\beta$-catenin and autophagy pathway through modulating the miR-619-5p/Pygo2 and miR-619-5p/ATG14 axes. Molecular cancer 2020, 19:118.

23. Sun S, Wu Y, Guo W, Yu F, Kong L, Ren Y, Wang Y, Yao X, Jing C, Zhang C: STAT3/HOTAIR signaling axis regulates HNSCC growth in an EZH2-dependent manner. Clinical Cancer Research 2018:clincanres.2248.2016.

24. Liu YR, Jiang YZ, Xu XE, Hu X, Shao ZM: Comprehensive Transcriptome Profiling Reveals Multigene Signatures in Triple-Negative Breast Cancer. Clinical Cancer Research An Official Journal of the American Association for Cancer Research 2016, 22:1653.

25. Rech AJ, Hannah D, Kotzin JJ, Jorge HM, Minn AJ, Christina TSV, Vonderheide RH: Radiotherapy and CD40 Activation Separately Augment Immunity to Checkpoint Blockade in Cancer. Cancer Research 2018, 78:4282.

26. Huang D, Chen J, Yang L, Ouyang Q, Li J, Lao L, Zhao J, Liu J, Lu Y, Xing Y, et al: NKILA IncRNA promotes tumor immune evasion by sensitizing $T$ cells to activation-induced cell death. Nature immunology 2018, 19:1112-1125.

27. Xu H, Jiang Y, Xu X, Su X, Liu Y, Ma Y, Zhao Y, Shen Z, Huang B, Cao X: Author Correction: Inducible degradation of IncRNA Sros1 promotes IFN-y-mediated activation of innate immune responses by stabilizing Stat1 mRNA. Nature immunology 2020, 21:477-478. 
28. Fu W, Lei C, Yu Y, Liu S, Li T, Lin F, Fan X, Shen Y, Ding M, Tang Y, et al: EGFR/Notch Antagonists Enhance the Response to Inhibitors of the PI3K-Akt Pathway by Decreasing Tumor-Initiating Cell Frequency. Clinical cancer research : an official journal of the American Association for Cancer Research 2019, 25:2835-2847.

29. Liu Q, Yu S, Zhao W, Qin S, Chu Q, Wu K: EGFR-TKls resistance via EGFR-independent signaling pathways. Molecular cancer 2018, 17:53.

30. Gong C, Maquat LE: IncRNAs transactivate STAU1-mediated mRNA decay by duplexing with 3' UTRs via Alu elements. Nature 2011, 470:284-288.

31. Kim Y, Furic L, Desgroseillers L, Maquat L: Mammalian Staufen1 recruits Upf1 to specific mRNA 3'UTRs so as to elicit mRNA decay. Cell 2005, 120:195-208.

32. Zhao W, Yang Y, Song L, Kang T, Du T, Wu Y, Xiong M, Luo L, Long J, Men K: A Vesicular Stomatitis Virus-Inspired DNA Nanocomplex for Ovarian Cancer Therapy. Advanced Science 2018:1700263.

33. Indocyanine green binds to DOTAP liposomes for enhanced optical properties and tumor photoablation. Biomaterials Science 2019, 7.

34. Esposito R, Bosch N, Lanzós A, Polidori T, Pulido-Quetglas C, Johnson R: Hacking the Cancer Genome: Profiling Therapeutically Actionable Long Non-coding RNAs Using CRISPR-Cas 9 Screening. Cancer Cell 2019.

35. Federico C, Elisabetta M, Luca CG, Stefania B, Elisa R, Vienna L, Vanesa G, Claudia L, Alessandra C, Stefania D: Akt Phosphorylation and Gefitinib Efficacy in Patients With Advanced Non-Small-Cell Lung Cancer. Journal of the National Cancer Institute 2004:462-463.

36. Gawronski AR, Michael U, Zhang Y, Yen-Yi L, Niknafs YS, Ramnarine VR, Rohit M, Feng F, Chinnaiyan AM, Collins CC: MechRNA: prediction of IncRNA mechanisms from RNA-RNA and RNA-protein interactions. Bioinformatics 2018:18.

37. Bonasio R, Shiekhattar R: Regulation of transcription by long noncoding RNAs. Annual review of genetics 2014, 48:433-455.

38. Luo H, Zhu G, Xu J, Lai Q, Huang S: HOTTIP IncRNA Promotes Hematopoietic Stem Cell Self-Renewal Leading to AML-like Disease in Mice. Cancer Cell 2019, 36.

39. Wu Y, Zhang L, Kim D, Liu X, Lee D, Yang J, Ahn M, Vansteenkiste J, Su W, Felip E, et al: Phase Ib/II Study of Capmatinib (INC280) Plus Gefitinib After Failure of Epidermal Growth Factor Receptor (EGFR) Inhibitor Therapy in Patients With EGFR-Mutated, MET Factor-Dysregulated Non-Small-Cell Lung Cancer. Journal of clinical oncology : official journal of the American Society of Clinical Oncology 2018, 36:3101-3109.

40. Soria J, Wu Y, Nakagawa K, Kim S, Yang J, Ahn M, Wang J, Yang J, Lu Y, Atagi S, et al: Gefitinib plus chemotherapy versus placebo plus chemotherapy in EGFR-mutation-positive non-small-cell lung cancer after progression on first-line gefitinib (IMPRESS): a phase 3 randomised trial. The Lancet Oncology 2015, 16:990-998.

41. Di H, Jianing C, Linbin Y, Qian O, Jiaqian L, Liyan L, Jinghua Z, Jiang L, Yiwen L, Yue X: NKILA IncRNA promotes tumor immune evasion by sensitizing $T$ cells to activation-induced cell death. 
Nature Immunology 2018.

42. Ma MZ, Li CX, Zhang Y, Weng MZ, Zhang... M-d: Long non-coding RNA HOTAIR, a c-Myc activated driver of malignancy, negatively regulates miRNA-130a in gallbladder cancer. Molecular Cancer 2014, 13.

43. Poli V, Fagnocchi L, Fasciani A, Cherubini A, Mazzoleni S, Ferrillo S, Miluzio A, Gaudioso G, Vaira V, Turdo A: MYC-driven epigenetic reprogramming favors the onset of tumorigenesis by inducing a stem cell-like state. Nature Communications 2018, 9:1024.

44. Casey SC, Baylot V, Felsher DW: The MYC Oncogene is a Global Regulator of the Immune Response. Blood 2018:blood-2017-2011-742577.

45. Pelengaris S, Khan M, Evan G: c-MYC: more than just a matter of life and death. Nature reviews Cancer 2002, 2:764-776.

46. Secombe J, Pierce S, Eisenman R: Myc: a weapon of mass destruction. Cell 2004, 117:153-156.

47. Herold S, Herkert B, Eilers M: Facilitating replication under stress: an oncogenic function of MYC? Nature reviews Cancer 2009, 9:441-444.

48. Zhao N, Cao J, Xu L, Tang Q, Dobrolecki LE, Lv X, Talukdar M, Lu Y, Wang X, Hu DZ: Pharmacological targeting of MYC-regulated IRE1/XBP1 pathway suppresses MYC-driven breast cancer. Journal of Clinical Investigation 2018, 128:1283-1299.

49. Al Saleh K, Safwat R, Bedair A, Al Basmy A, Hooda HS, Shete J: Phase II/III randomized study of hyperfractionated radiotherapy with concomitant cetuximab versus concomitant chemotherapy in advanced nonmetastatic head and neck cancer: Preliminary report. Journal of Clinical Oncology 2012, 30:-

50. Tan EH, Goh C, Lim WT, Soo KC, Khoo ML, Tan T, Tan DSW, Ang MK, Ng QS, Tan PH: Gefitinib, cisplatin, and concurrent radiotherapy for locally advanced head and neck cancer: EGFR FISH, protein expression, and mutational status are not predictive biomarkers. Annals of Oncology 2012, 23:1010-1016.

51. Munster PN, Aggarwal R, Hong D, Schellens JHM, Van dN, R., Specht J, Witteveen PO, Werner T, Dees EC, Bergsland EK: First-in-human phase i study of GSK2126458, an oral pan-class i phosphatidylinositol-3-kinase inhibitor, in patients with advanced solid tumor malignancies. Clinical Cancer Research An Official Journal of the American Association for Cancer Research 2015:1932.

52. Hu Q, Ye Y, Chan LC, Li Y, Yang L: Oncogenic IncRNA downregulates cancer cell antigen presentation and intrinsic tumor suppression. Nature Immunology 2019, 20:1.

53. Jänne PA, Cohen RB, Laird AD, Macé S, Engelman JA, Ruiz-Soto R, Rockich K, Xu J, Shapiro GI, Martinez P: Phase I Safety and Pharmacokinetic Study of the PI3K/mTOR Inhibitor SAR245409 (XL765) in Combination with Erlotinib in Patients with Advanced Solid Tumors. Journal of Thoracic Oncology 2014.

54. Zhao C, Miao J, Shen G, Li J, Shi M, Zhang N, Hu G, Chen X, Hu X, Wu S: Anti-Epidermal Growth Factor Receptor (EGFR) Monoclonal Antibody combined with Cisplatin and 5-Fluorouracil in Patients 
with Metastatic Nasopharyngeal Carcinoma after Radical Radiotherapy: A Multicentre, Open-label, Phase II Clinical Trial. Annals of Oncology 2019.

55. Burris H, Flinn I, Patel M, Fenske T, Deng C, Brander D, Gutierrez M, Essell J, Kuhn J, Miskin H, et al: Umbralisib, a novel PI3Kס and casein kinase-1 $\varepsilon$ inhibitor, in relapsed or refractory chronic lymphocytic leukaemia and lymphoma: an open-label, phase 1, dose-escalation, first-in-human study. The Lancet Oncology 2018, 19:486-496.

56. Sarker D, Ang J, Baird R, Kristeleit R, Shah K, Moreno V, Clarke P, Raynaud F, Levy G, Ware J, et al: First-in-human phase I study of pictilisib (GDC-0941), a potent pan-class I phosphatidylinositol-3kinase (PI3K) inhibitor, in patients with advanced solid tumors. Clinical cancer research : an official journal of the American Association for Cancer Research 2015, 21:77-86.

57. Inaba K, Oda K, Aoki K, Sone K, Ikeda Y, Miyasaka A, Kashiyama T, Fukuda T, Makii C, Arimoto T, et al: Synergistic antitumor effects of combination PI3K/mTOR and MEK inhibition (SAR245409 and pimasertib) in mucinous ovarian carcinoma cells by fluorescence resonance energy transfer imaging. Oncotarget 2016, 7:29577-29591.

58. Arend R, Davis A, Chimiczewski P, O'Malley D, Provencher D, Vergote I, Ghamande S, Birrer M: EMR 20006-012: A phase II randomized double-blind placebo controlled trial comparing the combination of pimasertib (MEK inhibitor) with SAR245409 (PI3K inhibitor) to pimasertib alone in patients with previously treated unresectable borderline or low grade ovarian cancer. Gynecologic oncology 2020, 156:301-307.

59. Nogueira-Rodrigues A, Moralez G, Grazziotin R, Carmo CC, Small IA, Alves FVG, Mamede M, Erlich F, Viegas C, Triginelli SA: Phase 2 trial of erlotinib combined with cisplatin and radiotherapy in patients with locally advanced cervical cancer. Cancer 2014, 120:1187-1193.

60. Li C, Liang G, Yao W, Sui J, Shen X, Zhang Y, Peng H, Hong W, Ye Y, Zhang Z, et al: Integrated analysis of long non-coding RNA competing interactions reveals the potential role in progression of human gastric cancer. International journal of oncology 2016, 48:1965-1976.

61. Engelman J, Zejnullahu K, Mitsudomi T, Song Y, Hyland C, Park J, Lindeman N, Gale C, Zhao X, Christensen $\mathrm{J}$, et al: MET amplification leads to gefitinib resistance in lung cancer by activating ERBB3 signaling. Science (New York, NY) 2007, 316:1039-1043.

62. Gautschi O, Menon R, Bertrand M, Murer C, Diebold J: Capmatinib and Osimertinib Combination Therapy for EGFR-Mutant Lung Adenocarcinoma. Journal of thoracic oncology : official publication of the International Association for the Study of Lung Cancer 2020, 15:e13-e15.

63. Yue D, Xu S, Wang Q, Li X, Shen Y, Zhao H, Chen C, Mao W, Liu W, Liu J, et al: Erlotinib versus vinorelbine plus cisplatin as adjuvant therapy in Chinese patients with stage IIIA EGFR mutationpositive non-small-cell lung cancer (EVAN): a randomised, open-label, phase 2 trial. The Lancet Respiratory medicine 2018, 6:863-873.

64. Guttman M, Rinn J: Modular regulatory principles of large non-coding RNAs. Nature 2012, 482:339346.

65. Lau E: Non-coding RNA: zooming in on IncRNA functions. Nature Reviews Genetics 2014, 15. 
66. Slack FJ, Chinnaiyan AM: The Role of Non-coding RNAs in Oncology. Cell 2019, 179:1033-1055.

67. Teplyuk NM, Uhlmann EJ, Gabriely G, Volfovsky N, Wang Y, Teng J, Karmali P, Marcusson E, Peter M, Mohan A: Therapeutic potential of targeting microRNA-10b in established intracranial glioblastoma: first steps toward the clinic. EMBO Molecular Medicine 2016, 8:268-287.

68. Manabe S, Oshita F, Murakami S, Kondo T, Yamada K: Phase II study of carboplatin and pemetrexed followed by gefitinib for patients with advanced non-small cell lung cancer harboring sensitive EGFR mutation. Journal of Clinical Oncology 2016, 34:e20581-e20581.

69. Rikova K, Guo A, Zeng Q, Possemato A, Yu J, Haack H, Nardone J, Lee K, Reeves C, Li Y, et al: Global survey of phosphotyrosine signaling identifies oncogenic kinases in lung cancer. Cel/2007, 131:1190-1203.

70. Costa D, Schumer S, Tenen D, Kobayashi S: Differential responses to erlotinib in epidermal growth factor receptor (EGFR)-mutated lung cancers with acquired resistance to gefitinib carrying the L747S or T790M secondary mutations. Journal of clinical oncology : official journal of the American Society of Clinical Oncology 2008, 26:1182-1184; author reply 1184-1186.

71. Martinelli E, Ciardiello D, Martini G, Troiani T, Cardone C, Vitiello P, Normanno N, Rachiglio A, Maiello E, Latiano T, et al: Implementing anti-epidermal growth factor receptor (EGFR) therapy in metastatic colorectal cancer: challenges and future perspectives. Annals of oncology : official journal of the European Society for Medical Oncology 2020, 31:30-40.

72. Elkabets M, Pazarentzos E, Juric D, Sheng Q, Pelossof R, Brook S, Benzaken A, Rodon J, Morse N, Yan $\mathrm{J}$, et al: AXL mediates resistance to PI3Ka inhibition by activating the EGFR/PKC/mTOR axis in head and neck and esophageal squamous cell carcinomas. Cancer cel/ 2015, 27:533-546.

73. He L, Liu X, Yang J, Li W, Liu S, Liu X, Yang Z, Ren J, Wang Y, Shan L, et al: Imbalance of the reciprocally inhibitory loop between the ubiquitin-specific protease USP43 and EGFR/PI3K/AKT drives breast carcinogenesis. Cell research 2018, 28:934-951.

74. Bregni G, Sciallero S, Sobrero A: HER2 Amplification and Anti-EGFR Sensitivity in Advanced Colorectal Cancer. JAMA oncology 2019, 5:605-606.

75. Wheeler D, Dunn E, Harari P: Understanding resistance to EGFR inhibitors-impact on future treatment strategies. Nature reviews Clinical oncology 2010, 7:493-507.

76. Robichaux J, Elamin Y, Vijayan R, Nilsson M, Hu L, He J, Zhang F, Pisegna M, Poteete A, Sun H, et al: Pan-Cancer Landscape and Analysis of ERBB2 Mutations Identifies Poziotinib as a Clinically Active Inhibitor and Enhancer of T-DM1 Activity. Cancer cell 2019, 36:444-457.e447.

77. Baselga J, Arteaga C: Critical update and emerging trends in epidermal growth factor receptor targeting in cancer. Journal of clinical oncology : official journal of the American Society of Clinical Oncology 2005, 23:2445-2459.

\section{Figures}



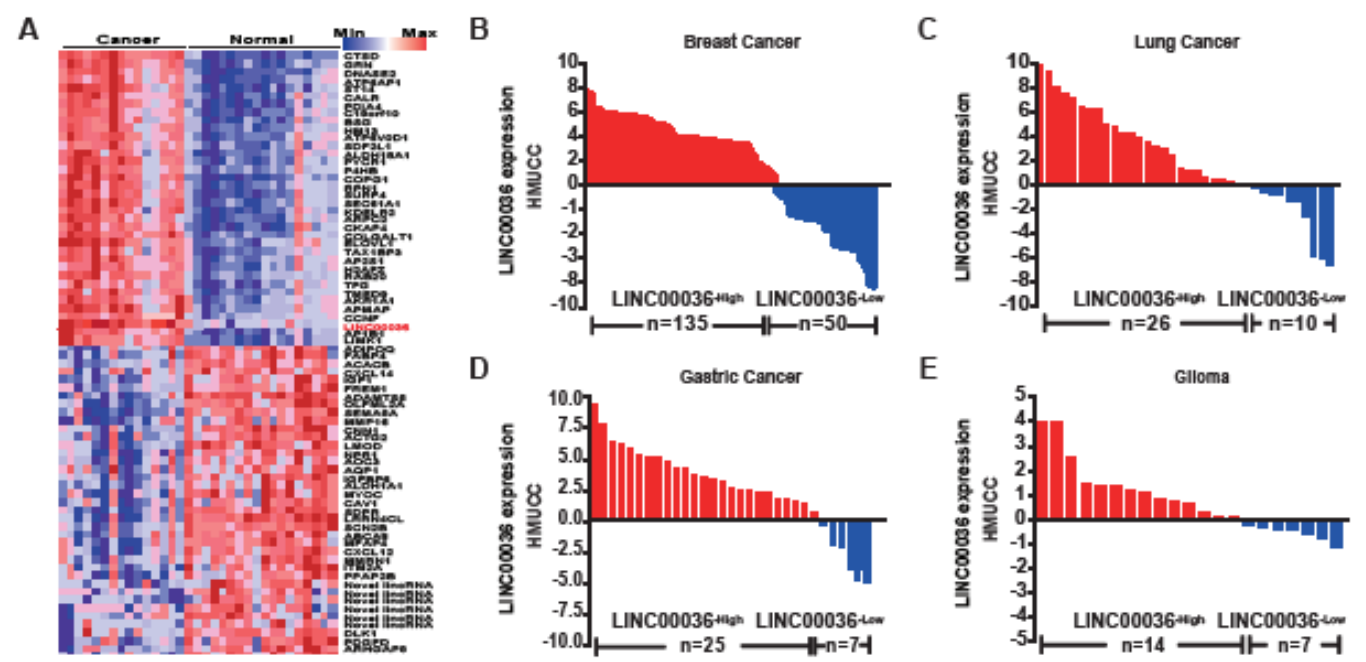

F
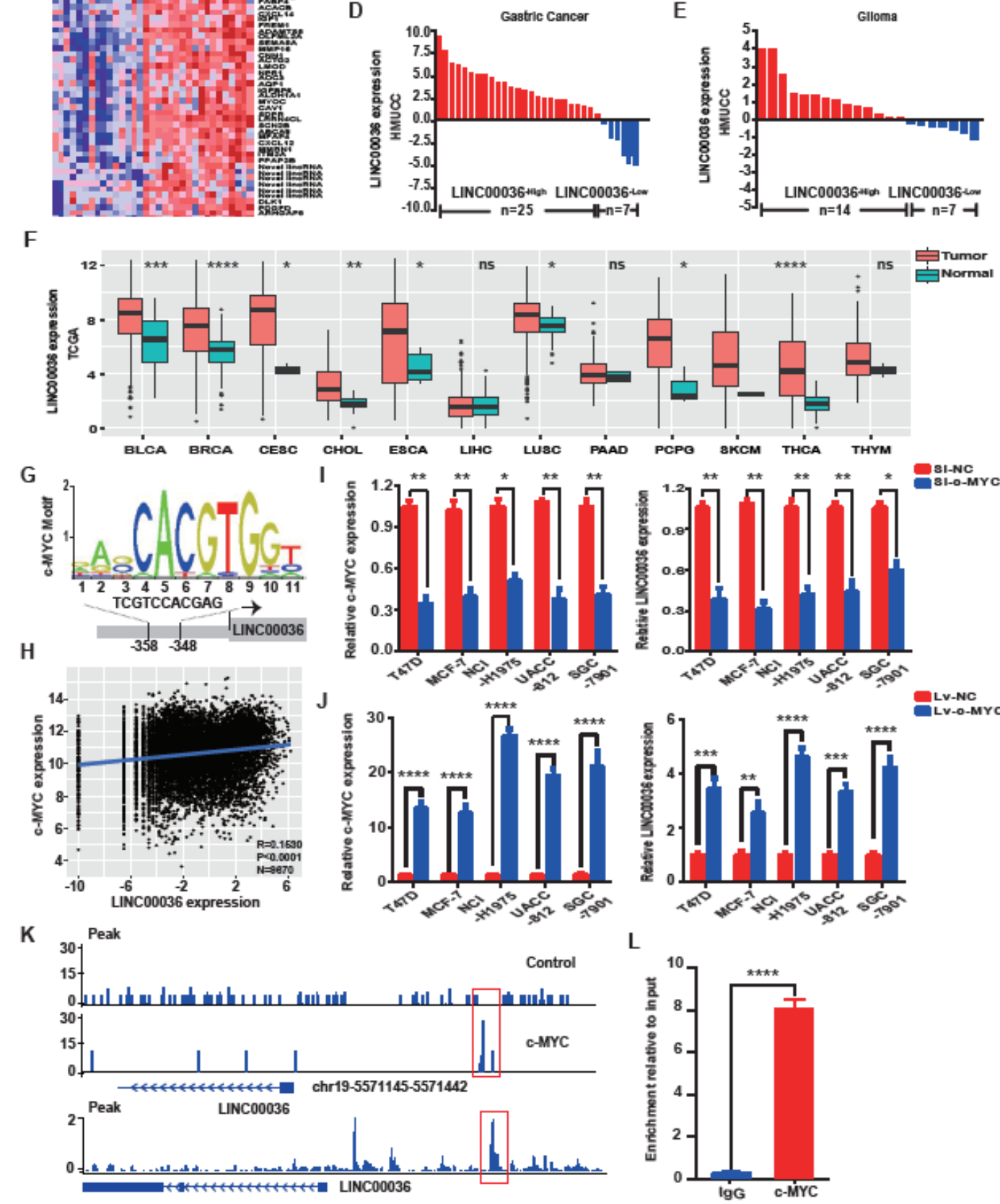

\section{Figure 1}

LINC00036 is overexpressed and is a c-MYC inducible IncRNA in human cancer. a. Heatmap of the whole transcriptomes of 30 breast specimens ( 15 breast cancer tissues and 15 adjacent normal tissues, and 3 normal breast tissues). Colors correspond to the expression level indicated by the log2-transformed scale bar to the right of the matrix. Red and blue reflect Max and Min levels, respectively. b-e. qRT-PCR analysis of LINC00036 expression in 185 breast cancer samples, 36 lung cancer samples, 21 glioma samples and 
32 gastric cancer samples in the HMUCC cohort. f. Expression of LINC00036 in different types of cancer in TCGA data (including BLCA, BRCA, CESC, CHOL, ESCA, LUSC, PCPG, SKCM and THCA). g. Putative CMYC-binding site in the transcriptional start of LINC00036 predicted in the JASPAR database. $h$. Correlation between c-MYC and LINC00036 expression in 9,670 patients in 33 types of cancer from TCGA data. i. Efficiency of c-MYC knockdown (left) and LINC00036 expression in UACC-812, T47D, MCF-7, NCIH1975, and SGC-7901 cells with c-MYC knockdown (right). j. Efficiency of c-MYC overexpression (left) and LINC00036 expression in UACC-812, T47D, MCF-7, NCl-H1975, and SGC-7901 cells with c-MYC knockdown (right). k. Enrichment peak of c-MYC in the promoter at chr19-5571145-5571442 of LINC00036 in ChIP-seq of UACC-812 cells (upper panel). Enrichment peak of c-MYC in the promoter of LINC00036 in ChIP-seq of MCF-7 cells in ENCODE database (lower panel). I. ChIP-PCR showed the binding of c-MYC to the LINC00036 promoter. The data are presented as the mean $\pm S D$, ${ }^{P}<0.05$; $* * P<$ $0.01 ; * \star * \mathrm{P}<0.001 ; * \star \star \star \mathrm{P}<0.0001$. 
A
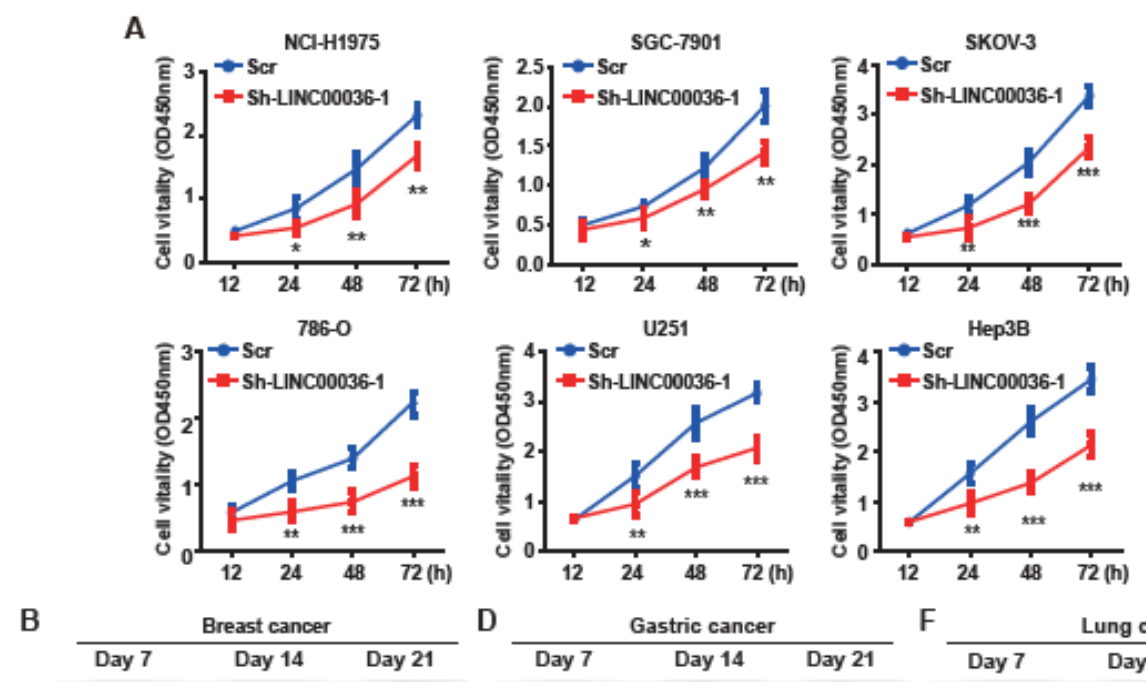

\begin{tabular}{lcc} 
D & \multicolumn{3}{c}{ Gastric cancer } \\
\cline { 2 - 3 } Day 7 & Day 14 & Day 21
\end{tabular}

\begin{tabular}{lll} 
& \multicolumn{3}{c}{ Lung cancer } \\
\cline { 2 - 4 } 7 & Day 14 & Day 21
\end{tabular}
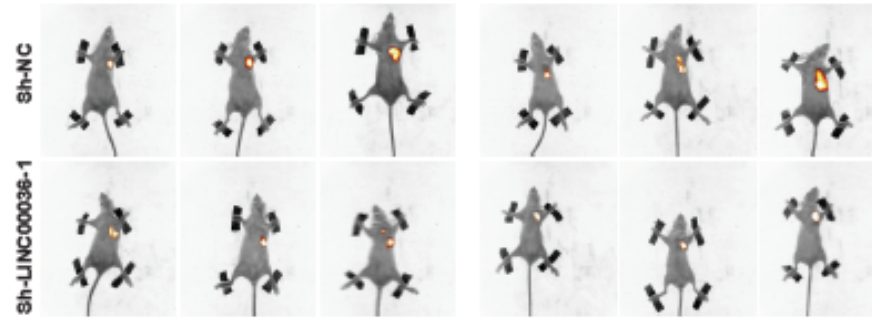

$\mathrm{C}$
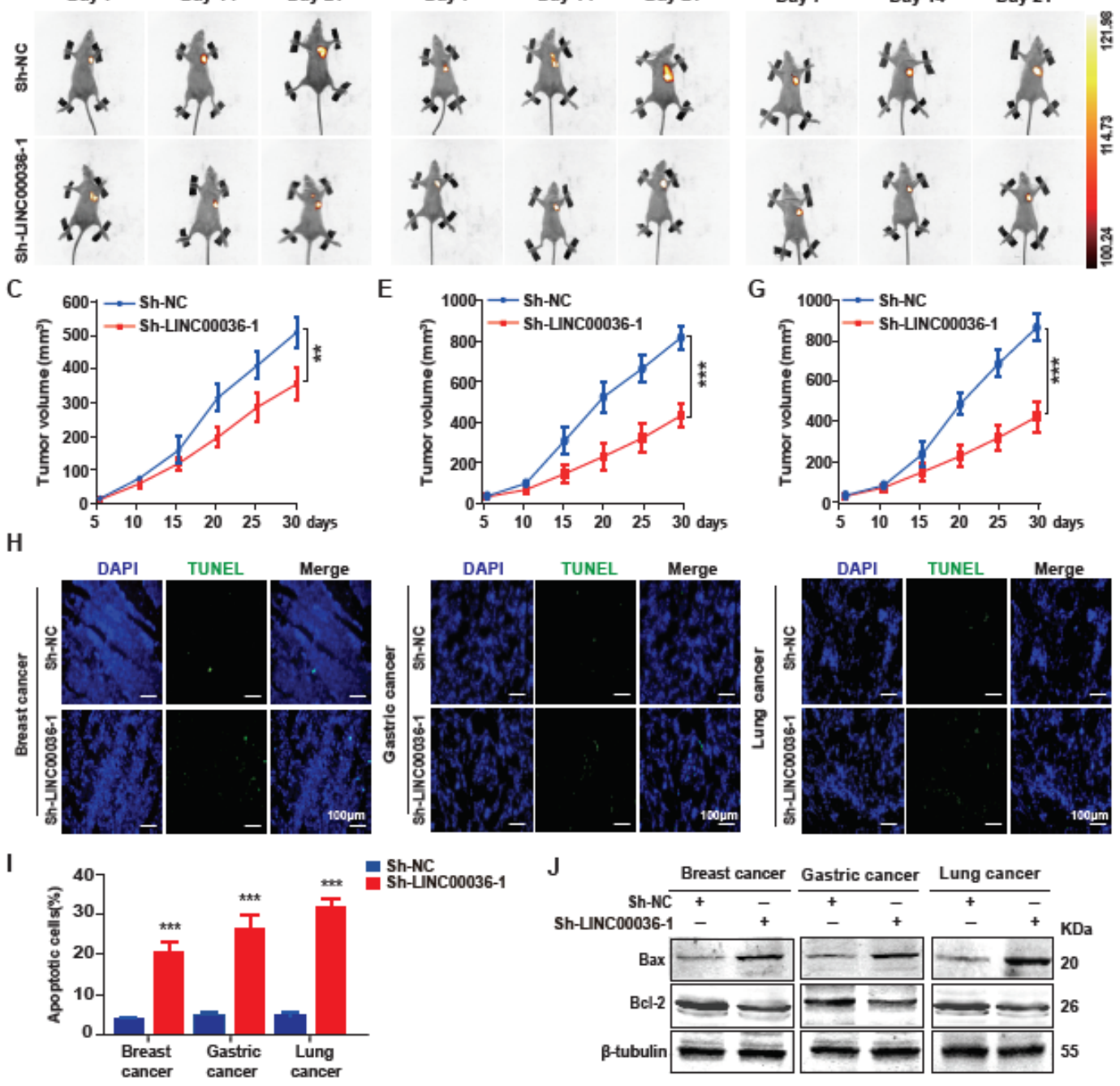

I
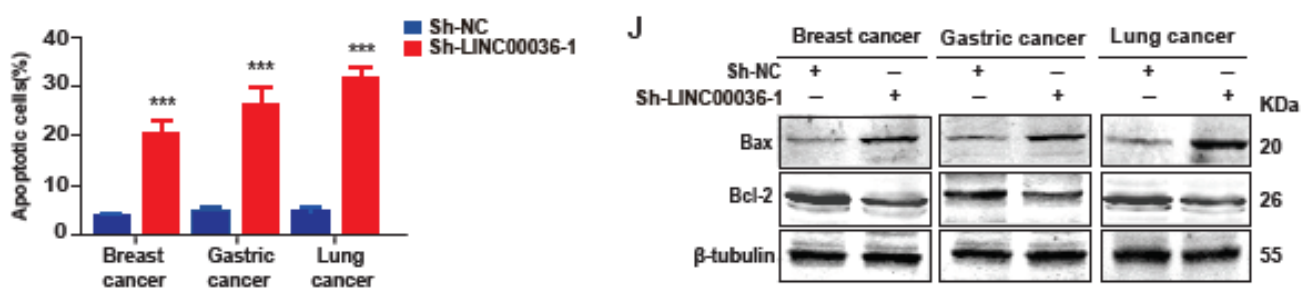

\section{Figure 2}

LINC00036 acts as an oncogenic IncRNA in human cancer. a. CCK-8 assays for LINC00036 knockdown and scramble group in $\mathrm{NCl}-\mathrm{H} 1975$ cells (lung cancer cell line), SGC-7901 cells (gastric cancer cell line), SKOV-3 cells (ovarian cancer cell line), 786-0 cells (kidney cancer cell line), U251 cells (glioma cell line) and Hep3B cells (liver cancer cell line). b-g. Tumor growth in sh-LINC00036 group and the scramble group in breast cancer (UACC-812)(b and c), gastric cancer (SGC-7901)(d and e), and lung cancer (NCI-H1975)(f 
and $\mathrm{g}$ ) models ( $\mathrm{n}=5$ mice per group). $\mathrm{h}$ and $\mathrm{i}$. Apoptosis in sh-LINC00036 group and scramble group in the above three cancer cell lines using TUNEL assay. (Original magnification: $200 x$; scale bar: $100 \mu \mathrm{m}$ ). j. Western blot analysis of Bax and Bcl-2 expression in the sh-LINC00036 group and scramble group in the above three cancer cell line models. The data are presented as the mean $\pm S D, * * P<0.01 ; * \star * P<0.001$.

A

B
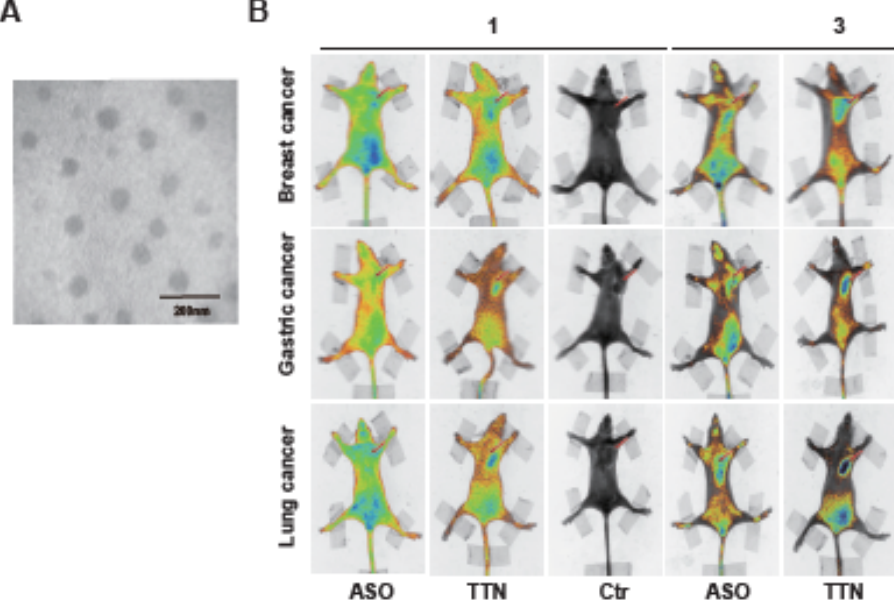

C

D
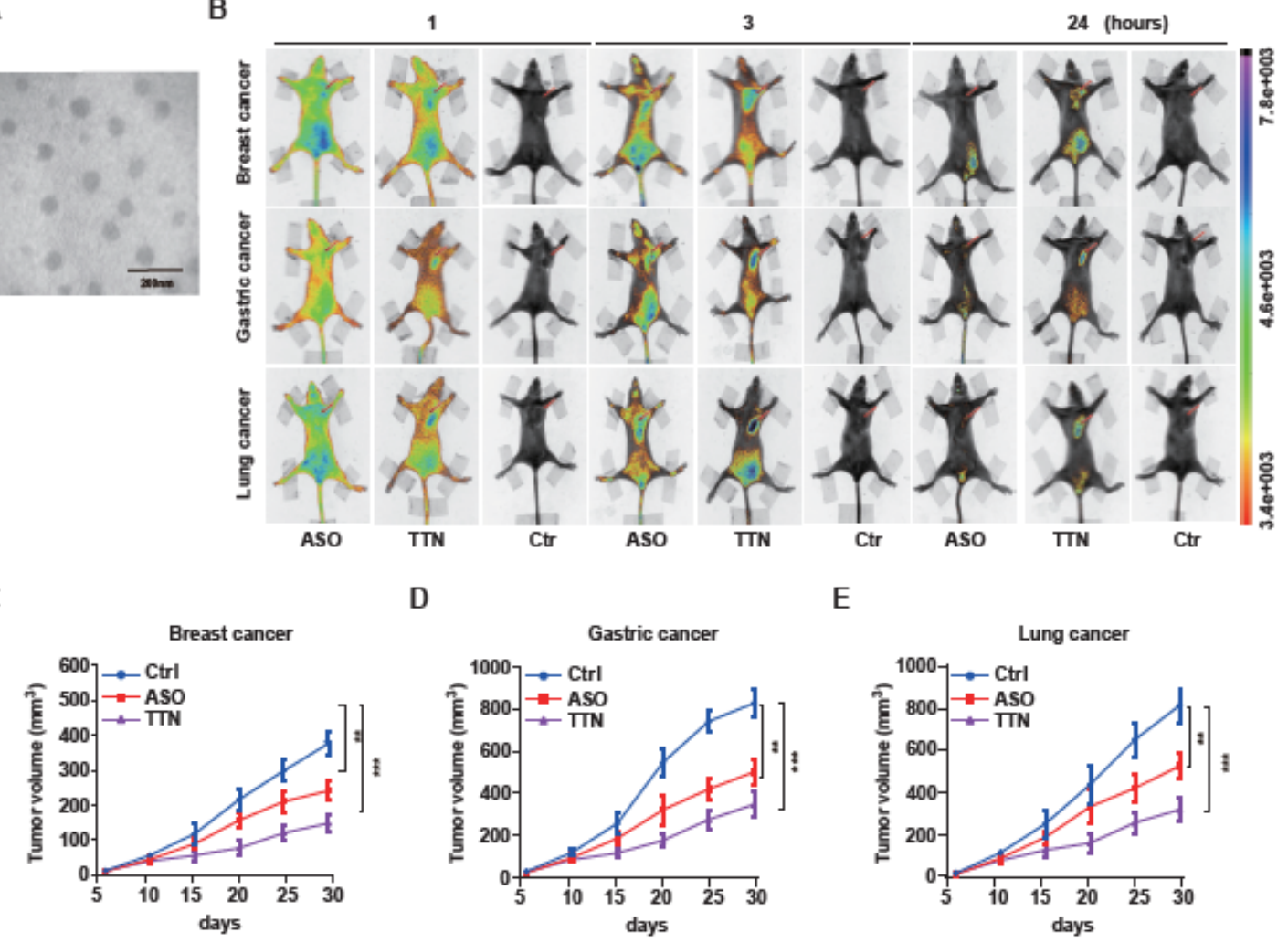

$\mathrm{E}$

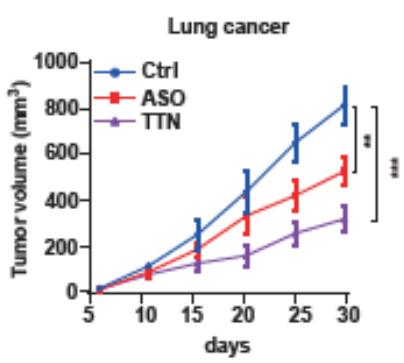

F

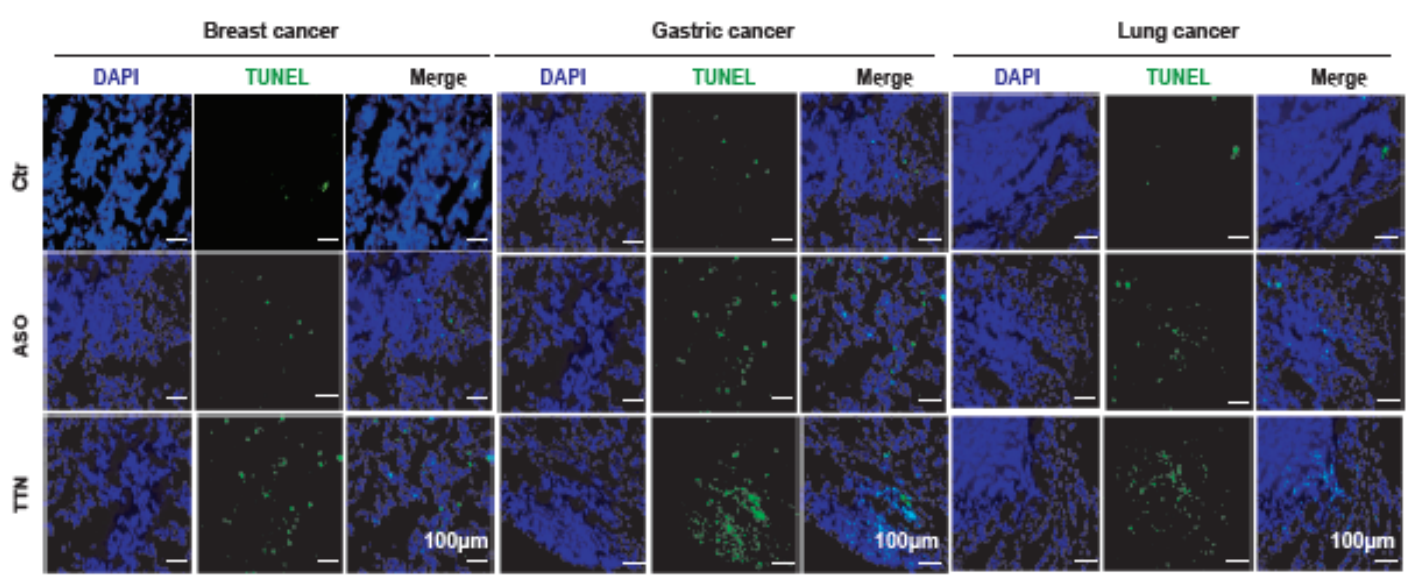

G
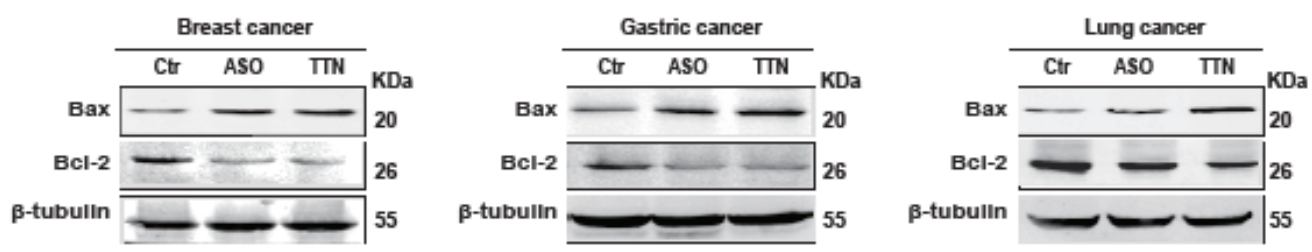

Figure 3 
LINC00036 is a promising novel therapeutic target. a. Transmission electron microscopy image of TTN. (Electron microscopy. 8000x. Scale bar: $200 \mu \mathrm{m}$ ). b. TTN showed higher tumor accumulation and retention than that of ASO alone in breast cancer UACC-812, gastric cancer SGC-7901 and lung cancer $\mathrm{NCl}-\mathrm{H} 1975$ tumor mice models ( $\mathrm{n}=4$ mice per group). c-e. Representative ASO treatment tumor volumes in (c)breast cancer (UACC-812), (d)gastric cancer (SGC-7901), and (e)lung cancer (NCl-H1975) models in the three treatment groups (control, ASO, and TTN) ( $n=4$ mice per group). $f$. TUNEL assays for liver, kidney, heart, lung, spleen and skin treated with different treatments. (Original magnification. $200 \times$. Scale bar: $100 \mu \mathrm{m})$. g. Western blot analysis for Bax and Bcl-2 expression in the TTN, ASO, or control group for the above three cancer cell line xenografts. The data are presented as the mean $\pm S D, * \star P<0.01 ; * \star \star P<$ 0.001 . 
A

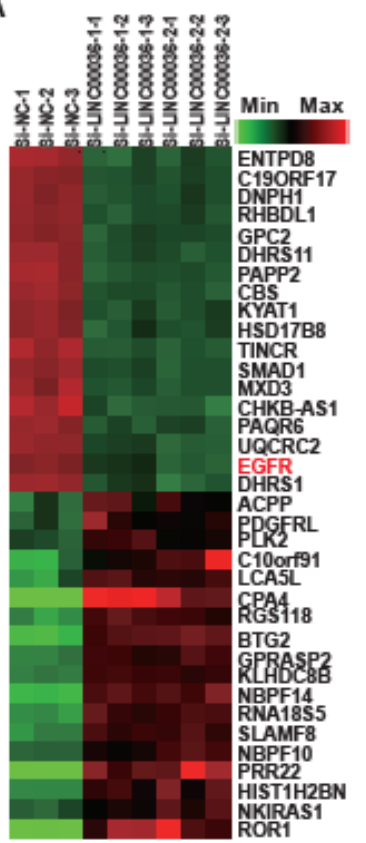

B

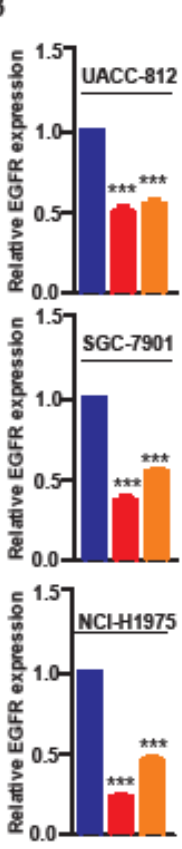

D

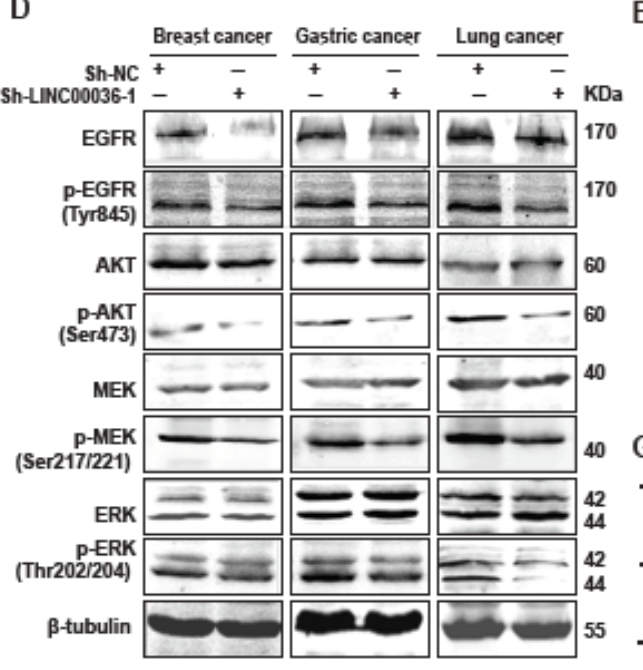

H

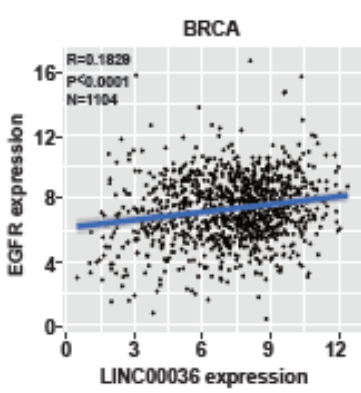

C

S1-LINC00036-1

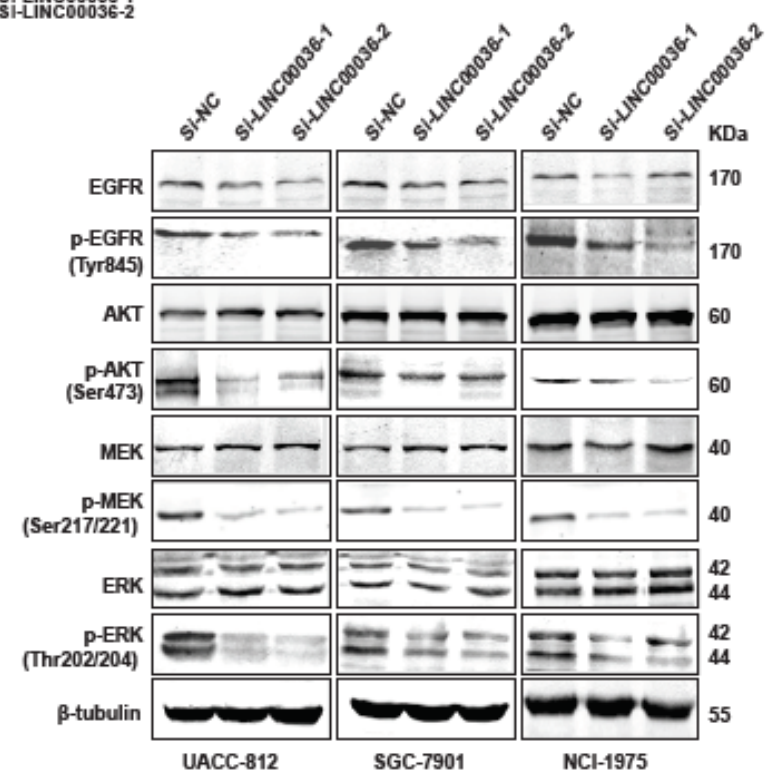

$\mathrm{E}$
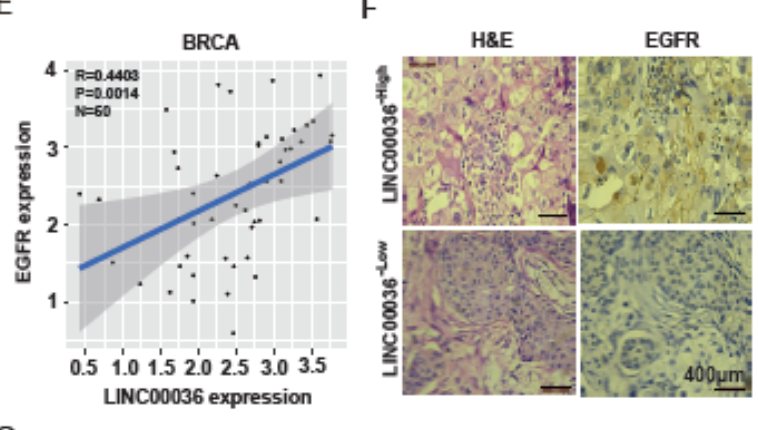
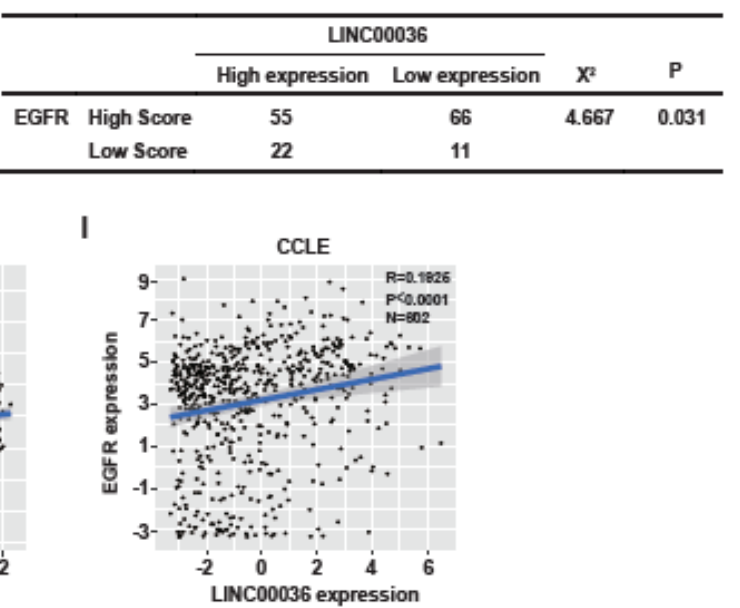

Figure 4

LINC00036 ignites the PI3K/AKT signaling pathway and MAPK signaling pathway through up-regulation EGFR expression. a. Heatmap of BGISEQ-500 RNA-seq of UACC-812 cells with or without LINC00036 knockdown. Colors correspond to the expression levels indicated by the log2-transformed scale bar to the right of the matrix. Red and green colors reflect Max and Min levels, respectively. b. Expression of EGFR mRNA in UACC-812, SGC-7901, and NCl-1975 cells with LINC00036 knockdown. c. Protein expression of 
EGFR, p-EGFR, p-AKT, p-MEK, and p-ERK in different treatment groups in UACC-812, SGC-7901, and NClH1975 cells in vitro. d. Protein expression of EGFR and p-EGFR, p-AKT, p-MEK, and p-ERK in different treatment groups in UACC-812, SGC-7901, and NCI-H1975 cells in vivo. e. LINC00036 expression correlated positively with EGFR mRNA expression in 50 samples from patients with breast cancer in the HMUCC cohort. $f$ and g. EGFR protein level in LINC00036-high and LINC00036-low breast cancer tissues from 154 patients in the HMUCC cohort. X2 test for their expression correlation. (Scale bar, $400 \times \mu \mathrm{m}$ ). $\mathrm{h}$ and i. Correlation of LINC00036 and EGFR expression in TCGA BRCA(h) and CCLE data(i). The data are presented as the mean $\pm S D$, $\star \star \star ~ P<0.001$. 
A

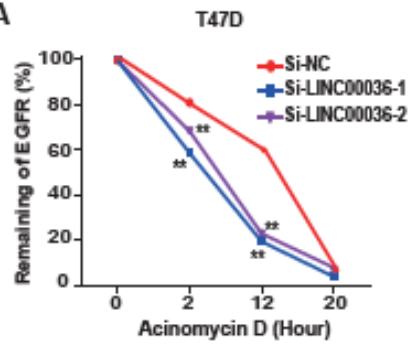

D

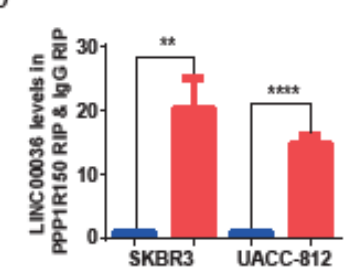

G

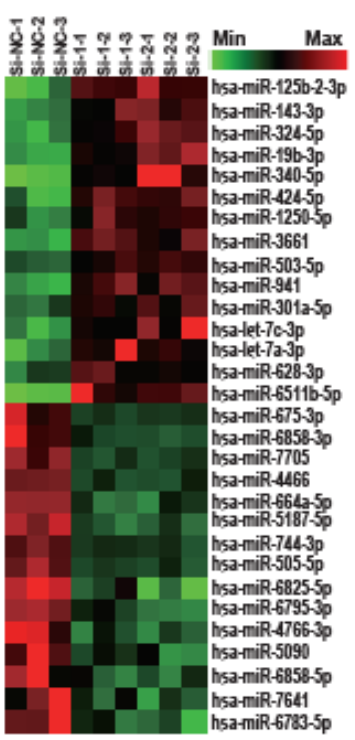

B

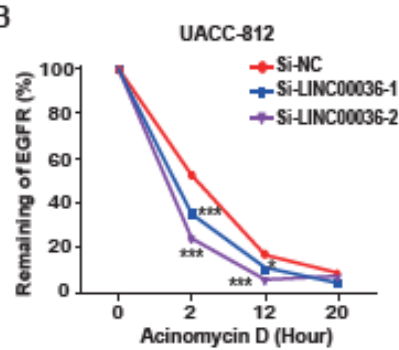

E

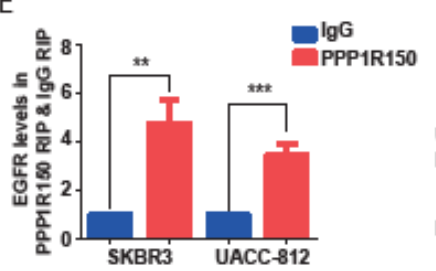

H
C

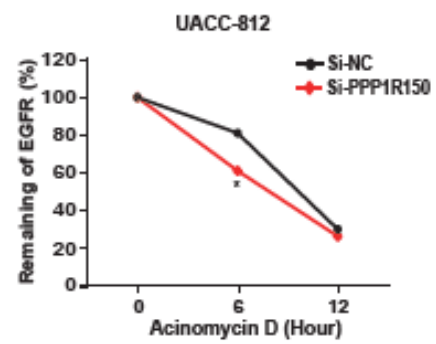

F

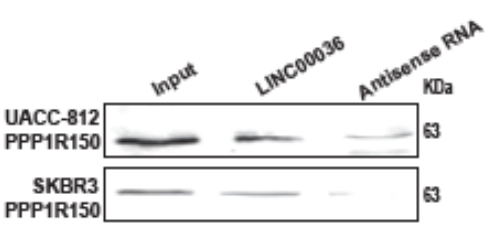

SiNC

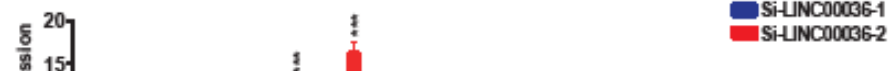

I
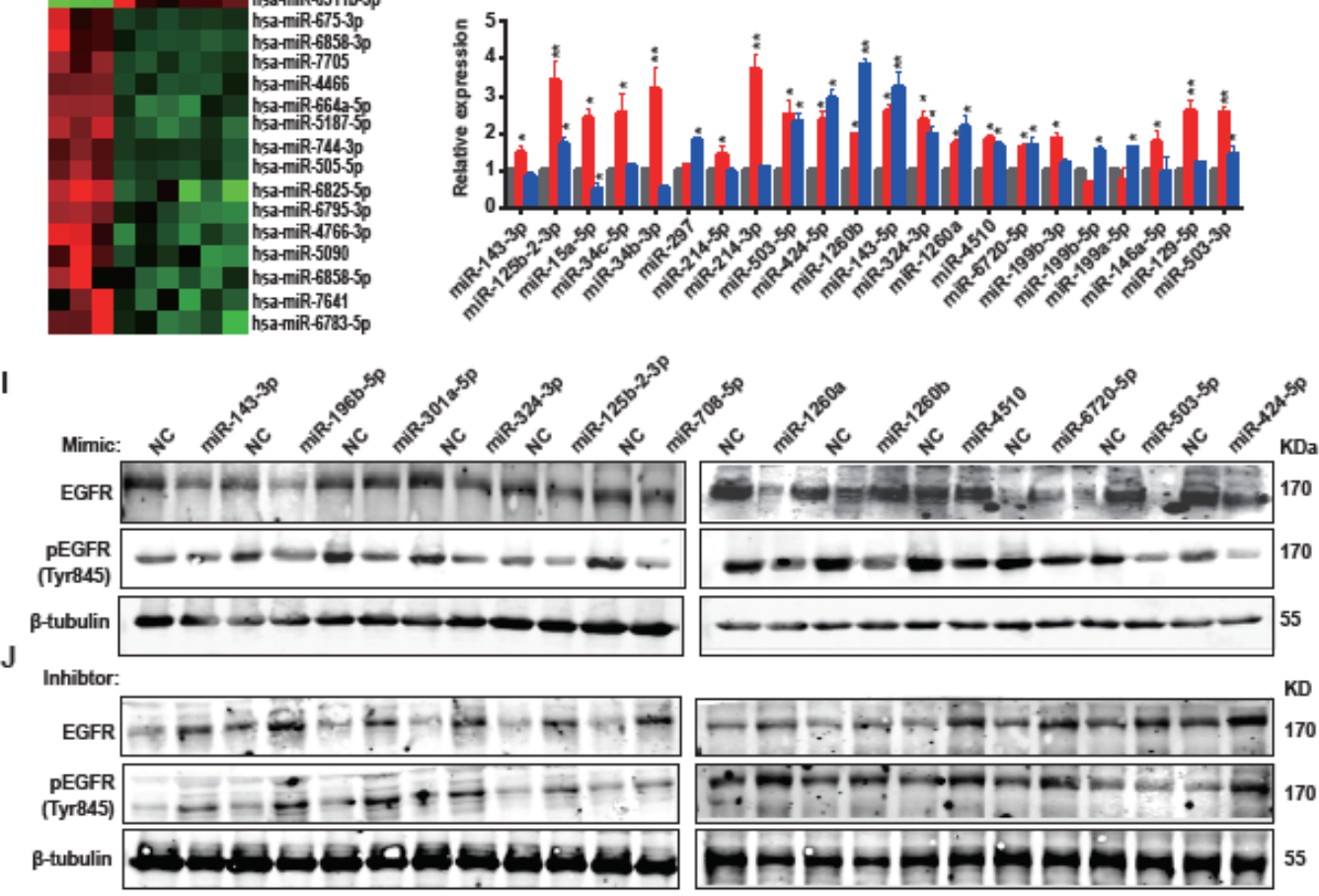

Figure 5

LINC00036 stabilizes EGFR expression via RNA-protein interaction and actes as a competing endogenous RNA (ceRNA). $a$ and b. The half-life of the EGFR mRNA in LINC00036 knockdown T47D (a) and UACC-812 cells (b) treated with $5 \mu \mathrm{M}$ actinomycin D. c. The half-life of the EGFR mRNA in PPP1R150 knockdown UACC-812 cells treated with $5 \mu \mathrm{M}$ actinomycin D. $\mathrm{d}$ and e. Enrichment of LINC00036 and EGFR in PPP1R150 group and IgG group in UACC-812 and SKBR3 cells using RIP assays. IgG was the negative 
control. f. Enrichment of PPP1R150 in the LINC00036 group and control in UACC-812 and SKBR3 cells via RNA pull-down assay. Antisense RNA acted as the negative control. g. Heatmap of BGISEQ-500 small RNA-seq in LINC00036 knockdown group and control group in T47D cells. Colors correspond to the expression level indicated by the log2-transformed scale bar to the right of the matrix. Red and green colors reflect Max and Min levels, respectively. h. qRT-PCR validation of upregulated microRNAs in the LINC00036 knockdown group in T47D. i and j. Western blot analysis for EGFR and p-EGFR protein levels in UACC-812 cells treated with microRNA mimics (i) and inhibitors (j) of miR-143-3p, miR-196b-5p, miR301a-5p, miR-324-3p, miR-125b-2-3p, miR-1260a, miR-1260b, miR-4510, miR-6720-5p, and miR-503-5p,

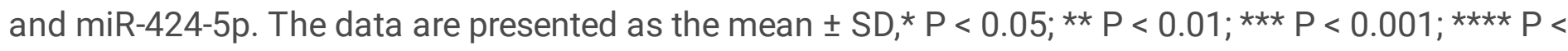
0.0001 . 
A

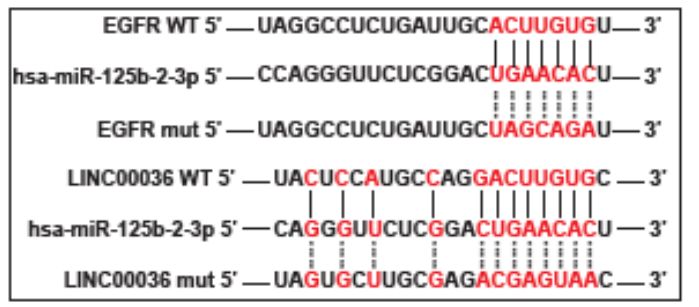

C

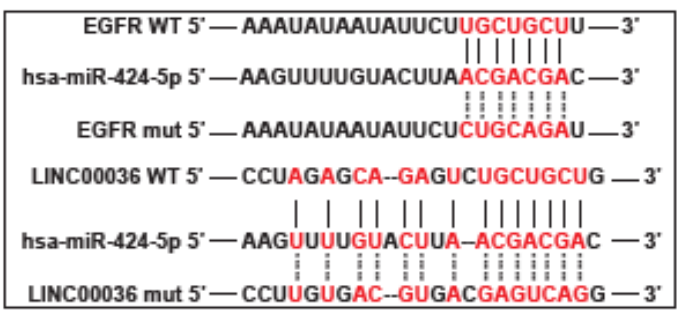

E

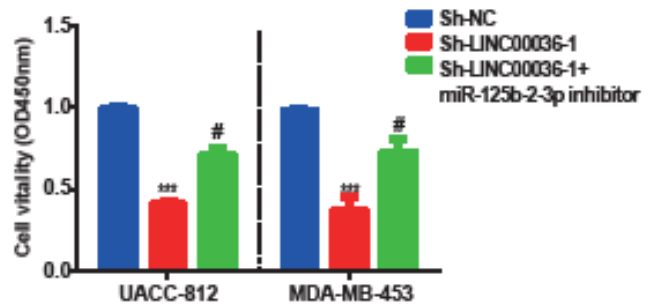

B
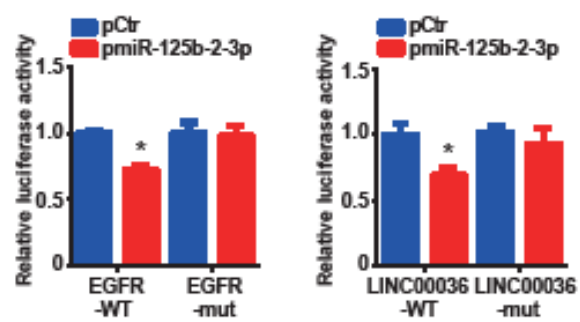

D
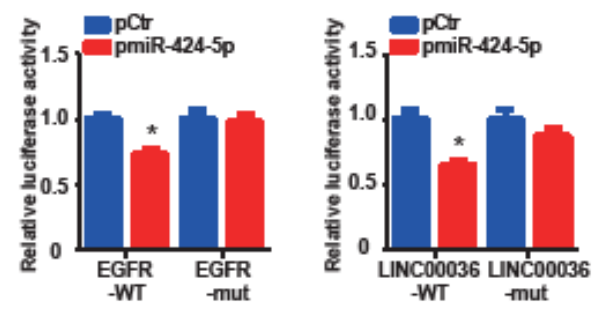

$\mathrm{F}$

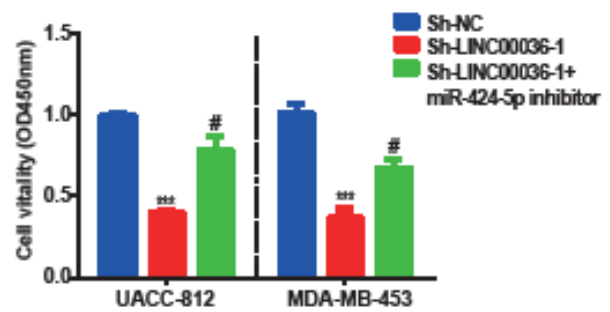

G

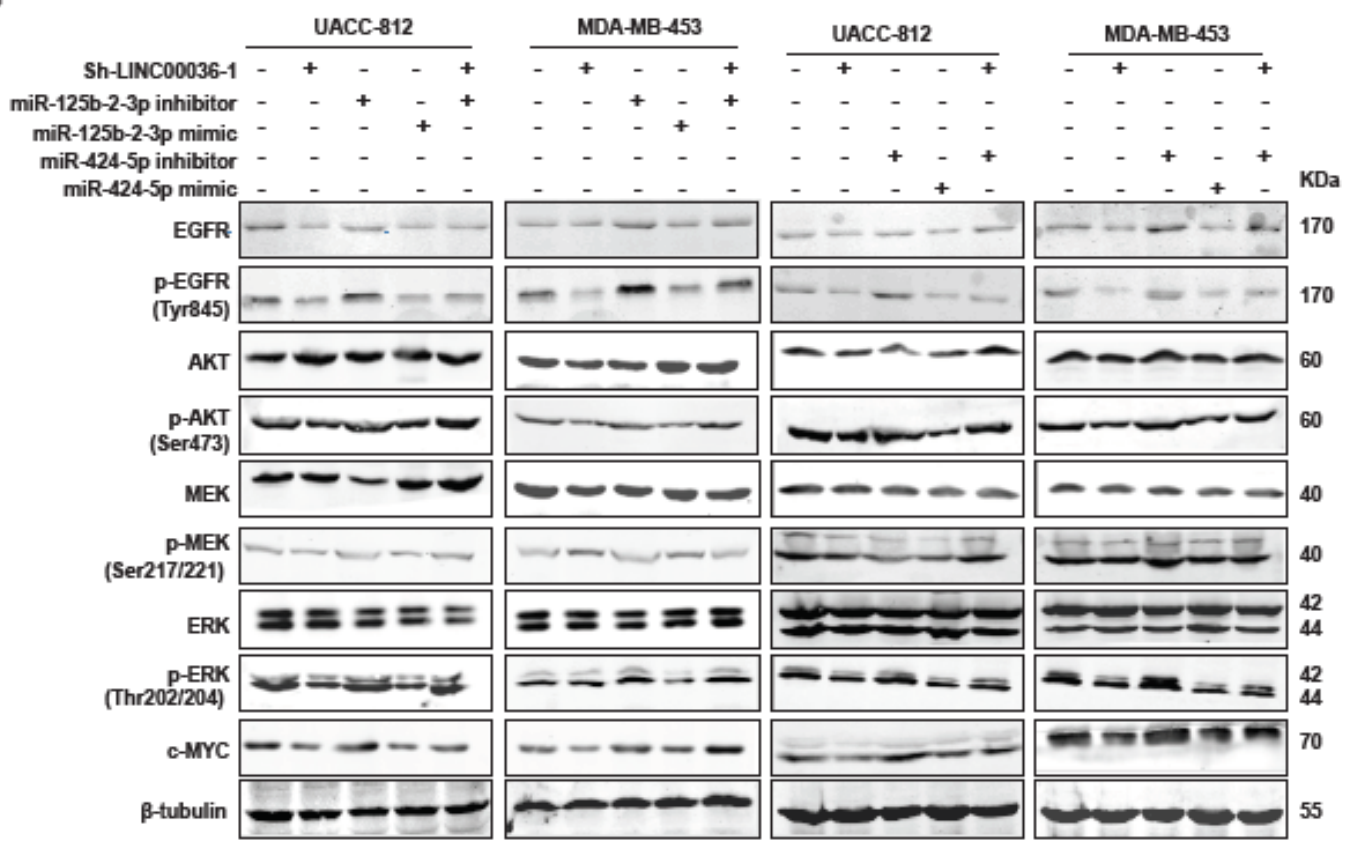

\section{Figure 6}

LINC00036 activates the EGFR pathway and its downstream targets as a ceRNA. a. Predicted wild type or mutated binding sites of miR-125b-2-3p in the 3' UTR of EGFR and LINC00036. b. Luciferase reporter assay for determining the interactions between miR-125b-2-3p and its wild type or mutated binding sites in the 3' UTR of EGFR and LINC00036 in HEK293T cells. c. Predicted binding sites or mutated binding sites of miR-424-5p in the 3' UTR of EGFR and LINC00036. d. Luciferase reporter assay for analyzing 
interactions between miR-424-5p and its wild type or mutated binding sites in the 3 ' UTR of EGFR and LINC00036 in HEK293T cells. e and f. Growth ability of UACC-812 and MDA-MB-453 cells after cotransfection with sh-LINC00036, miR-125b-2-3p inhibitor (e) and miR-424-5p inhibitor (f) as determined by CCK-8 assays. g Western blot analysis for EGFR, p-EGFR, p-ATK, p-MAPK, p-ERK, and c-MYC expression after LINC00036 knockdown and treatment with mimics and inhibitors for miR-125b-2-3p and miR-424-5p in UACC-812 and MDA-MB-453 cells. The data are presented as the mean $\pm S D$, $* * P<0.01 ; \star \star \star P<0.001$

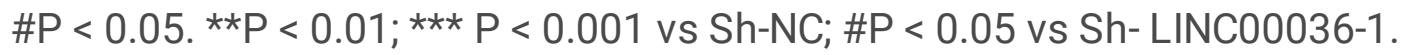

A
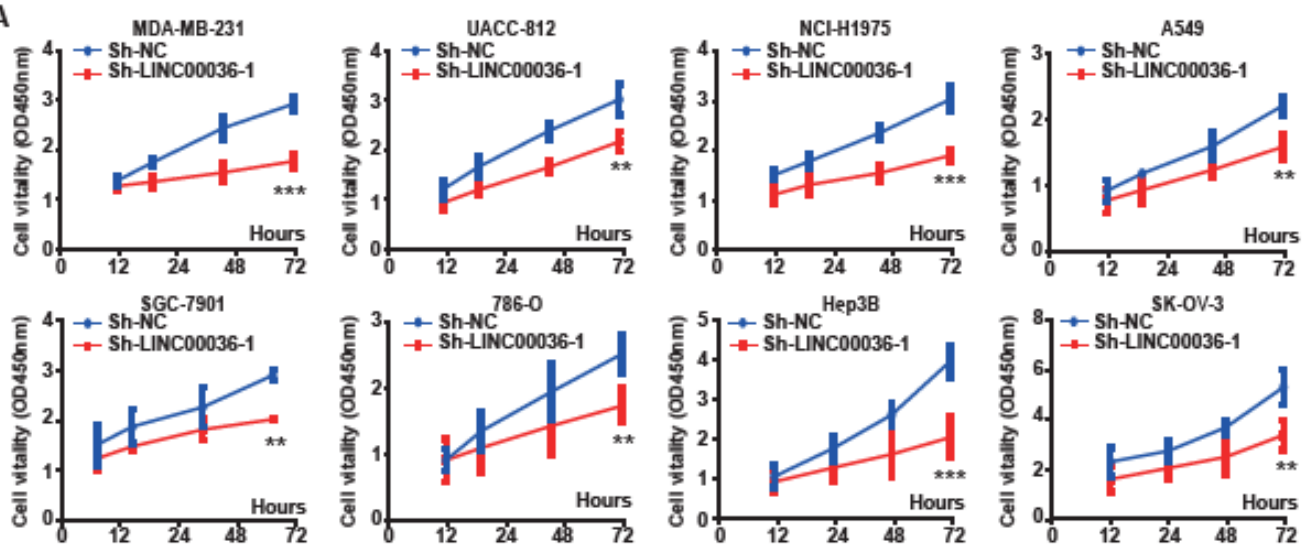

B

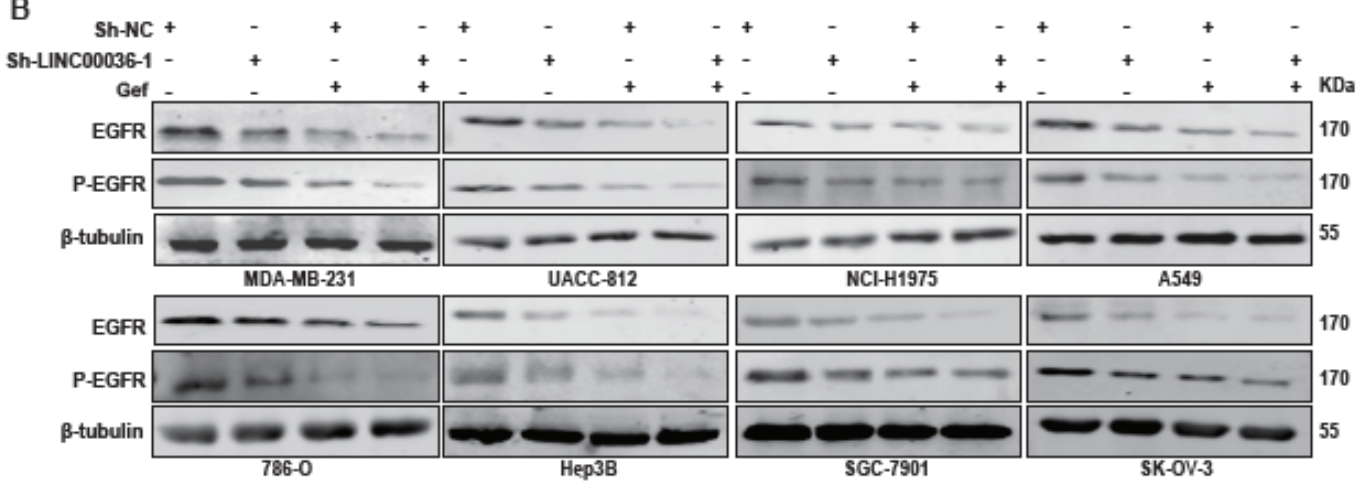

C
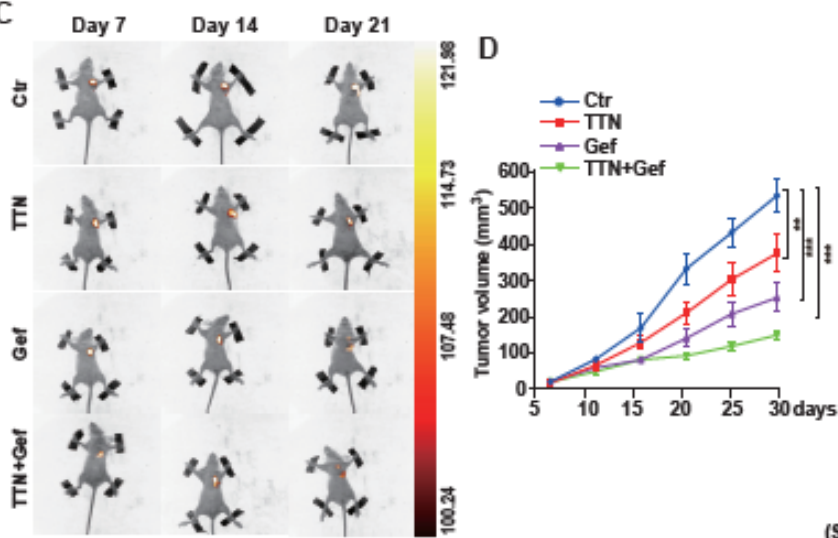

E

Ctr

TTN

Gef

TTN+Gef

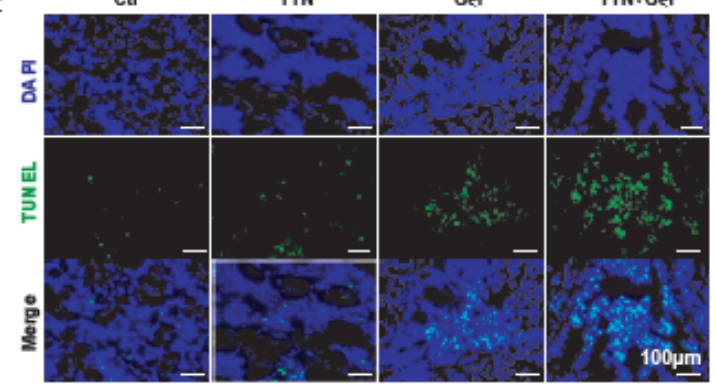

F

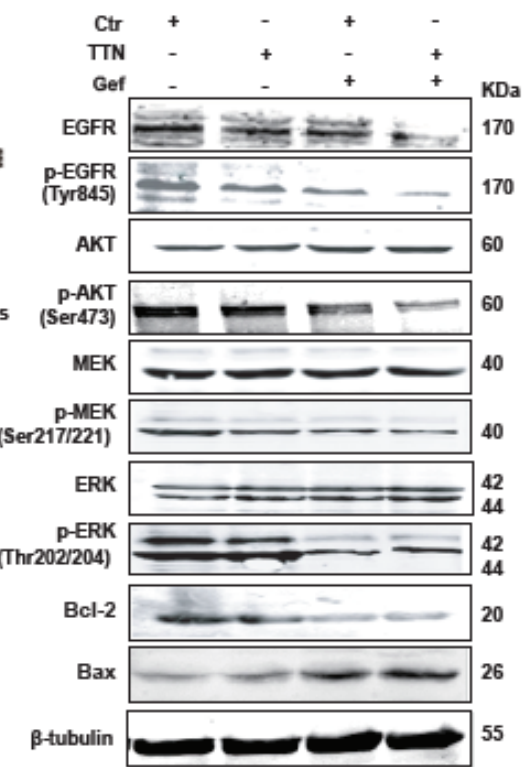




\section{Figure 7}

LINC00036 knockdown enhances human cancer cell sensitiity to gefitinib in vitro and in vivo. a. CCK8 assays were performed to determine the role of LINC00036 knockdown in MDA-MB-231, UACC-812, NCIH1975, A549, SGC-7901, 786-O, Hep3B and SKOV-3 cells treated with gefitinib. b. The expression of total EGFR and p-EGFR of LINC00036 knockdown in MDA-MB-231, UACC-812, NCI-H1975, A549, SGC-7901, 786-0, Hep3B and SKOV-3 cells treated with gefitinib for $48 \mathrm{~h}$ by western blot. $\mathrm{c}$ and $\mathrm{d}$. Tumor size in UACC-812 tumor-bearing mice treated with a combination of LINC00036 knockdown and gefitinib, LINC00036 knockdown, and gefitinib alone on days 7, 14, day 21 ( $n=5$ mice per group). e. Cell apoptosis in UACC-812 tumor-bearing mice treated with LINC00036 knockdown plus gefitinib, LINC00036 knockdown, and gefitinib alone using the TUNEL assay (Original magnification, 200 ×; scale bar: 100 $\mu \mathrm{m})$. f. Western blot analysis for total EGFR, p-EGFR, p-AKT, p-MEK, p-ERK, Bax, and Bcl-2 in LINC00036 knockdown plus gefitinib treatment, LINC00036 knockdown, or gefitinib alone groups in the above three cancer cell line xenografts. The data are presented as the mean $\pm S D$, ** $P<0.01 ; * \star \star P<0.001$. 
A

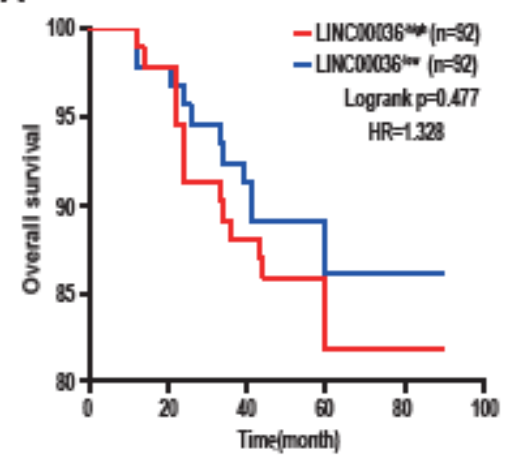

D

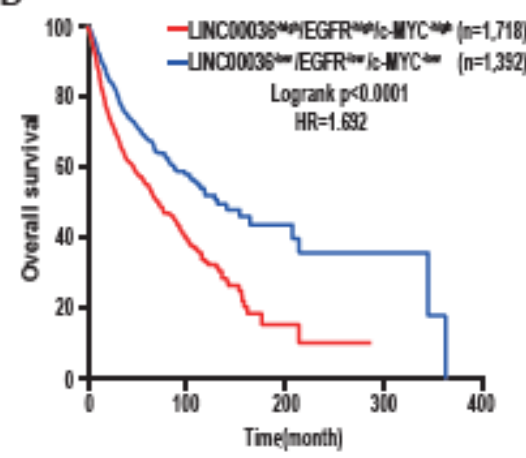

F

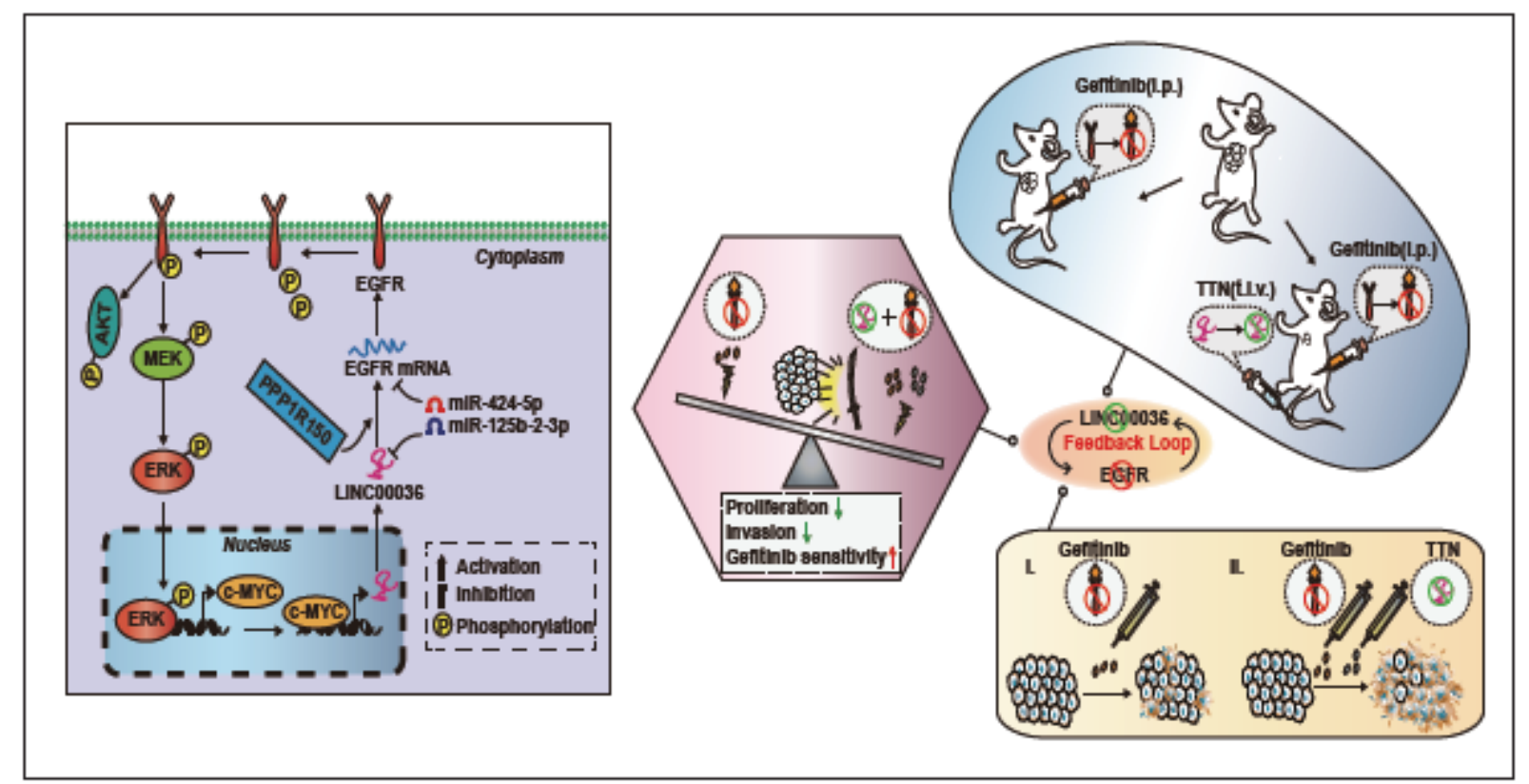

B

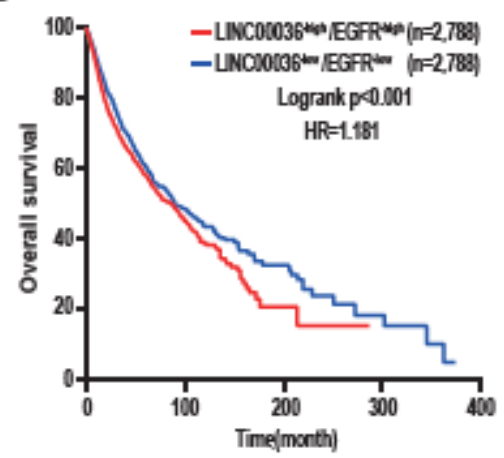

E

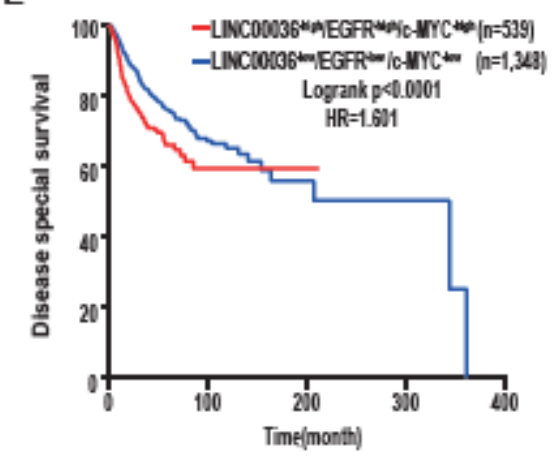

C

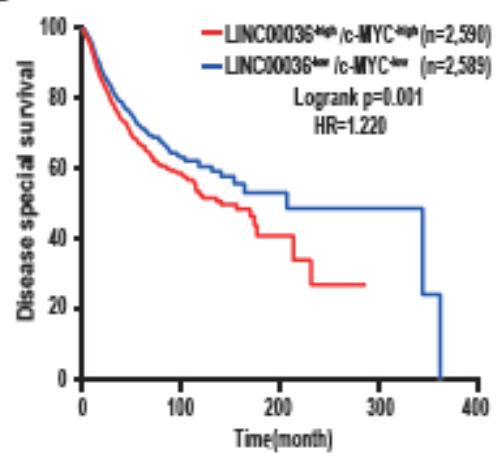


expression showed worse overall survival (d), disease special survival (e) than ones with LINC00036low/EGFR-low/c-MYC-low expression in above TCGA cohort. f. Schematic illustration depicting a proposed model of the molecular mechanism of LINC00036 and its roles in gefitinib sensitivity in human cancers. Positive feedback loop of the c-MYC-LINC00036-EGFR axis in human cancer (left panel) and cotargeting therapies by combining LINC00036 targeting and gefitinib reveals the synergistic anti-tumor effects in vivo and in vitro (right panel).

\section{Supplementary Files}

This is a list of supplementary files associated with this preprint. Click to download.

- Supplementaryinformation.docx

- FigureS1.pdf

- Figures2.pdf

- FigureS3.pdf

- Figures4.pdf

- Figures5.pdf

- FigureS6.pdf

- FigureS7.pdf

- SupplementaryTables1.docx

- SupplementaryTableS2.docx

- SupplementaryTableS3.docx

- SupplementaryTableS4.docx

- SupplementaryTableS5.docx

- SupplementaryTableS6.docx 